\title{
Taylor spectrum approach to Brownian-type operators with quasinormal entry
}

\author{
Sameer Chavan ${ }^{1} \cdot$ Zenon Jan Jabłoński $^{2}$ - II Bong Jung ${ }^{3} \cdot$ Jan Stochel $^{2}$
}

Received: 18 March 2020 / Accepted: 4 July 2020 / Published online: 9 August 2020

(c) The Author(s) 2020

\begin{abstract}
In this paper, we introduce operators that are represented by upper triangular $2 \times 2$ block matrices whose entries satisfy some algebraic constraints. We call them Brownian-type operators of class $\mathcal{Q}$, briefly operators of class $\mathcal{Q}$. These operators emerged from the study of Brownian isometries performed by Agler and Stankus via detailed analysis of the time shift operator of the modified Brownian motion process. It turns out that the class $\mathcal{Q}$ is closely related to the Cauchy dual subnormality problem which asks whether the Cauchy dual of a completely hyperexpansive operator is subnormal. Since the class $\mathcal{Q}$ is closed under the operation of taking the Cauchy dual, the problem itself becomes a part of a more general question of investigating subnormality in this class. This issue, along with the analysis of nonstandard moment problems, covers a large part of the paper. Using the Taylor spectrum technique culminates in a full characterization of subnormal operators of class $\mathcal{Q}$. As a consequence, we solve the Cauchy dual subnormality problem for expansive operators of class $\mathcal{Q}$ in the affirmative, showing that the original problem can surprisingly be extended to a class of operators that are far from being completely hyperexpansive. The Taylor spectrum approach turns out to be fruitful enough to allow us to characterize other classes of operators including $m$-isometries. We also study linear operator pencils associated with operators of class $\mathcal{Q}$ proving that the corresponding regions of subnormality are closed intervals with explicitly described endpoints.
\end{abstract}

Keywords Upper triangular $2 \times 2$ block matrix $\cdot$ Taylor's spectrum $\cdot$ Moment problems . Subnormal operator $\cdot m$-isometry $\cdot$ Linear operator pencil

Jan Stochel

Jan.Stochel@im.uj.edu.pl

Sameer Chavan

chavan@iitk.ac.in

Zenon Jan Jabłoński

Zenon.Jablonski@im.uj.edu.pl

Il Bong Jung

ibjung@knu.ac.kr

1 Department of Mathematics and Statistics, Indian Institute of Technology Kanpur, Kanpur, India

2 Instytut Matematyki, Uniwersytet Jagielloński, ul. Łojasiewicza 6, 30348 Kraków, Poland

3 Department of Mathematics, Kyungpook National University, Daegu 702-701, Korea 
Mathematics Subject Classification Primary 47B20 · 47B37 · Secondary 44A60

\section{Introduction}

Given two complex Hilbert spaces $\mathcal{H}$ and $\mathcal{K}$, we denote by $\boldsymbol{B}(\mathcal{H}, \mathcal{K})$ the Banach space of all bounded linear operators from $\mathcal{H}$ to $\mathcal{K}$. The kernel, the range, the adjoint and the modulus of an operator $T \in \boldsymbol{B}(\mathcal{H}, \mathcal{K})$ are denoted by $\mathscr{N}(T), \mathscr{R}(T), T^{*}$ and $|T|$, respectively. We regard $\boldsymbol{B}(\mathcal{H}):=\boldsymbol{B}(\mathcal{H}, \mathcal{H})$ as a $C^{*}$-algebra. The identity operator on $\mathcal{H}$ is denoted by $I_{\mathcal{H}}$, or simply by $I$ if no ambiguity arises. Recall that an operator $T \in \boldsymbol{B}(\mathcal{H})$ is said to be quasinormal if $T T^{*} T=T^{*} T T$, or equivalently if $T|T|=|T| T$. We say that $T$ is subnormal if there exist a complex Hilbert space $\mathcal{K}$ and a normal operator $N \in \boldsymbol{B}(\mathcal{K})$ such that $\mathcal{H} \subseteq \mathcal{K}$ (an isometric embedding) and $T h=N h$ for all $h \in \mathcal{H}$. It is well known that quasinormal operators are subnormal (see [19, Proposition II.1.7]). We refer the reader to [19] for more information on these classes of operators.

Let $T \in \boldsymbol{B}(\mathcal{H})$. We say that $T$ is a 2-isometry if $T^{* 2} T^{2}-2 T^{*} T+I=0$. We call $T$ a Brownian isometry if $T$ is a 2-isometry such that $\Delta_{T} \Delta_{T^{*}} \Delta_{T}=0$, where $\Delta_{T}=T^{*} T-I$. If $\Delta_{T} \geqslant 0$ and $\Delta_{T} T=\Delta_{T}^{1 / 2} T \Delta_{T}^{1 / 2}$, we say that $T$ is $\Delta_{T}$-regular. By a quasi-Brownian isometry we mean a $\Delta_{T}$-regular 2-isometry. It is well known that any 2 -isometry is left-invertible ${ }^{1}$ and $\Delta_{T} \geqslant 0$ ([42, Lemma 1]). The notion of a 2-isometry was invented by Agler in [2], while the notion of a Brownian isometry was introduced by Agler and Stankus in [3-5]. The class of 2-isometric operators emerged from the study of the time shift operator of the modified Brownian motion process from one side [3-5], and from the investigation of invariant subspaces of the Dirichlet shift from the other [42]. The class of $\Delta_{T}$-regular 2 -isometries were investigated in $[12,38]$ and in $[7,8]$ under the name of quasi-Brownian isometries.

Given a left-invertible operator $T \in \boldsymbol{B}(\mathcal{H})$, we set $T^{\prime}=T\left(T^{*} T\right)^{-1}$. Following [44], we call $T^{\prime}$ the Cauchy dual operator of $T$. Recall that if $T$ is left-invertible, then so is $T^{\prime}$ and $T=\left(T^{\prime}\right)^{\prime}$. Athavale noticed that the Cauchy dual operator of a completely hyperexpansive injective unilateral weighted shift is a subnormal contraction (see [11, Proposition 6] with $t=1$ ), but not conversely (see [11, Remark 4]). The Cauchy dual subnormality problem asks whether the Cauchy dual operator of a completely hyperexpansive operator (see Sect. 9 for the definition) is a subnormal contraction (see [17, Question 2.11]). As shown in [7], the answer is in the negative even for 2-isometries, that is, there are 2-isometries whose Cauchy dual operators are not subnormal (recall that each 2-isometry is completely hyperexpansive and that the Cauchy dual operator of a completely hyperexpansive operator is always a contraction). However, as proved in [7, Theorem 4.5], the Cauchy dual operator $T^{\prime}$ of a quasi-Brownian isometry $T$ is a subnormal contraction (see also [12, Theorem 3.4] for a recent generalization of this result to the case of completely hyperexpansive $\Delta_{T}$ -regular operators). This leads to the question of why this phenomenon can happen. We will try to answer it by regarding quasi-Brownian isometries as elements of a larger class of operators which is closed under the operation of taking the Cauchy dual (note that the class of quasi-Brownian isometries is not closed under this operation). As a consequence, in the larger class of operators, the Cauchy dual subnormality problem becomes a part of the more general question of finding necessary and sufficient conditions for subnormality.

$\overline{{ }^{1} \text { In this paper, left-invertibility and invertibility of an operator } T \in \boldsymbol{B}(\mathcal{H})}$ refer to the algebra $\boldsymbol{B}(\mathcal{H})$. 
Let us recall that nonisometric Brownian and quasi-Brownian isometries have upper triangular $2 \times 2$ block matrix representations with entries satisfying some algebraic constraints (see the remark just after Definition 1.1). For the purposes of our paper explained in the above discussion, we introduce a wider class of operators consisting of the so-called Brown-type operators.

Definition 1.1 We say that $T \in \boldsymbol{B}(\mathcal{H})$ is a Brownian-type operator if it has the block matrix form

$$
T=\left[\begin{array}{ll}
V & E \\
0 & Q
\end{array}\right]
$$

with respect to a nontrivial ${ }^{2}$ orthogonal decomposition $\mathcal{H}=\mathcal{H}_{1} \oplus \mathcal{H}_{2}$, where the operators $V \in \boldsymbol{B}\left(\mathcal{H}_{1}\right), E \in \boldsymbol{B}\left(\mathcal{H}_{2}, \mathcal{H}_{1}\right)$ and $Q \in \boldsymbol{B}\left(\mathcal{H}_{2}\right)$ satisfy the following conditions:

$$
\begin{gathered}
V \text { is an isometry, i.e., } V^{*} V=I, \\
V^{*} E=0, \\
Q E^{*} E=E^{*} E Q .
\end{gathered}
$$

Moreover, if

$$
Q \text { is quasinormal, }
$$

we call $T$ a Brownian-type operator of class $\mathcal{Q}$ and write $T=\left[\begin{array}{cc}V & E \\ 0 & Q\end{array}\right] \in \mathcal{Q}_{\mathcal{H}_{1}, \mathcal{H}_{2}}$; to simplify the terminology, we say that $T$ is an operator of class $\mathcal{Q}$. By analogy, if $Q$ is isometric (resp. unitary, normal, etc.), then $T$ is called an operator of class $\mathcal{I}$ (resp. $\mathcal{U}, \mathcal{N}$, etc.). If $\mathcal{K}$ is a complex Hilbert space and $\mathcal{H}=\mathcal{K} \oplus \mathcal{K}$ (understood as an external orthogonal sum), then we abbreviate $\mathcal{Q}_{\mathcal{K}, \mathcal{K}}$ to $\mathcal{Q}_{\mathcal{K}}$.

In Definition 1.1, we have decided to exclude the case when one of the summands $\mathcal{H}_{1}$ or $\mathcal{H}_{2}$ is absent because otherwise the operator $T$ is quasinormal. Moreover, by (2) and (3), the hypothesis that $E \neq 0$ excludes the case when $\mathcal{H}_{1}$ is finite-dimensional. Notice also that by the square root theorem [45, Theorem 2.4.4], the equality (4) is equivalent to $Q|E|=|E| Q$. One can deduce from [4, Proposition 5.37 and Theorem 5.48] (resp., [38, Proposition 5.1]) that a nonisometric operator $T \in \boldsymbol{B}(\mathcal{H})$ is a Brownian isometry (resp., a quasi-Brownian isometry) if and only if $T$ is of class $\mathcal{U}$ (resp., of class $\mathcal{I}$ ) (to avoid injectivity of $E$ postulated in [4, Proposition 5.37] and [38, Proposition 5.1], consult [7, Theorem 4.1]). This means that Brownian isometries are quasi-Brownian isometries. In view of [7, Example 4.4], the converse implication is not true in general.

It is worth pointing out that upper triangular $2 \times 2$ block matrices appear in different parts of operator theory and functional analysis on the occasion of investigating variety of topics; for example, the hyperinvariant subspace problem [24, 34-36], the Halmos similarity problem for polynomially bounded operators [26, 40], the task of finding models for the time shift operator for modified Brownian motion process [3-5], the question of characterizing invertibility of upper triangular $2 \times 2$ block matrices [31], the task of searching for a

\footnotetext{
${ }^{2}$ Nontriviality means that $\mathcal{H}_{1} \neq\{0\}$ and $\mathcal{H}_{2} \neq\{0\}$.
} 
Fig. 1 Spectral region for subnormality of operators of class $\mathcal{Q}$

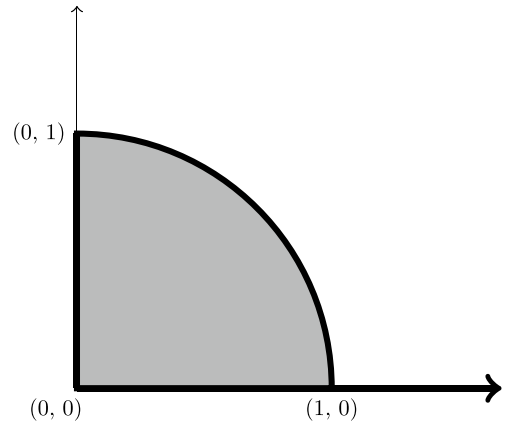

model theory for 2-hyponormal operators [22], the problem of determining a complete set of unitary invariants for the class of Cowen-Douglas operators realized as upper triangular $2 \times 2$ block matrices [33], and many others.

We state now the main result of this paper which characterizes subnormality of operators of class $\mathcal{Q}$ in terms of the Taylor spectrum $\sigma(|Q|,|E|)$ of the pair $(|Q|,|E|)$. The spectral region for subnormality of operators of class $\mathcal{Q}$ is described by Theorem 1.2(iii) and illustrated in Fig. 1. We refer the reader to Sect. 2 for the necessary definitions and notations.

Theorem 1.2 Suppose $T=\left[\begin{array}{ll}V & E \\ 0 & Q\end{array}\right] \in \mathcal{Q}_{\mathcal{H}_{1}, \mathcal{H}_{2}}$. Let $P \in \boldsymbol{B}\left(\mathcal{H}_{2}\right)$ be the orthogonal projection of $\mathcal{H}_{2}$ onto $\mathscr{M}:=\mathscr{R}(|E|)$. Then the operators $|Q|,|E|$ and $P$ commute, $\mathscr{M}$ reduces $|Q|$ and $|E|$, and the following conditions are equivalent:

(i) $T$ is subnormal,

(ii) $\sigma_{\sharp}(|Q|,|E|) \subseteq \overline{\mathbb{D}}_{+}$, where $\sigma_{\sharp}(|Q|,|E|):=\sigma(|Q|,|E|) \cap\left(\mathbb{R}_{+} \times(0, \infty)\right)$,

(iii) $\sigma(|Q|,|E|) \subseteq \overline{\mathbb{D}}_{+} \cup\left(\mathbb{R}_{+} \times\{0\}\right)$,

(iv) $(|Q| P,|E|)$ is a spherical contraction,

(v) $\left(\left.|Q|\right|_{\mathscr{M}},|E|_{\mathscr{M}}\right)$ is a spherical contraction,

(vi) $\sigma\left(\left.|Q|\right|_{\mathscr{M}},\left.|E|\right|_{\mathscr{M}}\right) \subseteq \overline{\mathbb{D}}_{+}$.

Moreover, if $T$ is subnormal, then

$$
\sigma(|Q|,|E|) \subseteq\left(\overline{\mathbb{D}}_{+} \cup\left(\mathbb{R}_{+} \times\{0\}\right)\right) \cap(\sigma(|Q|) \times \sigma(|E|)) .
$$

The proof of Theorem 1.2 is fairly long, and it occupies most of Sects. 3, 4 and 5. The theorem itself has many applications spread over Sects. 5, 6 and 10. In particular, we show that contractions of class $\mathcal{Q}$ are subnormal (see Corollary 5.2), we solve the Cauchy dual subnormality problem for expansive operators of class $\mathcal{Q}$ in the affirmative (see Corollary 6.2) and, what is more important, we completely characterize subnormality of the Cauchy-duals of left-invertible operators of class $\mathcal{Q}$ (see Theorem 6.1). The study of linear operator pencils associated with operators of class $\mathcal{Q}$ provides a useful test of the applicability of the main theorem (see Theorems 10.1 and 10.2).

The Taylor spectrum approach developed in this paper for the purpose of investigating subnormality turns out to be efficient when studying other collections of operators of class $\mathcal{Q}$ including $m$-contractions, $m$-isometries, etc. (see Sect. 9). In fact, it appears to be effective even in providing explicit formulas for the norm of operators of class $\mathcal{Q}$ (see (17) in Sect. 3) and for the right endpoints of the intervals of subnormality of linear operator pencils associated with operators of class $\mathcal{Q}$ (see (75) and (79) in Sect. 10). The 
Taylor spectrum technique is also applied to characterize quasi-Brownian and Brownian isometries of class $\mathcal{Q}$ in Sects. 7 and 8, respectively. Unexpectedly, the Brownian case is essentially more complicated. The reader has to be aware of the fact that quasi-Brownian (and so Brownian) isometries are always of class $\mathcal{Q}$, however relative to properly selected orthogonal decompositions (of the underlying Hilbert spaces), which are not necessarily easy to be determined in concrete cases.

We conclude Introduction by pointing out that the overwhelming majority of the characterizations of selected subclasses of the class $\mathcal{Q}$ that appear in this paper consist in finding for a given subclass a minimal universal subset of the Euclidean plane having the property that an operator $T=\left[\begin{array}{ll}V & E \\ 0 & Q\end{array}\right] \in \mathcal{Q}_{\mathcal{H}_{1}, \mathcal{H}_{2}}$ belongs to the subclass if and only if the Taylor spectrum $\sigma(|Q|,|E|)$ of the pair $(|Q|,|E|)$ is contained in the aforementioned subset. The universality of this subset lies in the fact that it does not depend on the choice of the orthogonal decomposition $\mathcal{H}_{1} \oplus \mathcal{H}_{2}$ of the underlying Hilbert space $\mathcal{H}$ relative to which a given operator $T \in \boldsymbol{B}(\mathcal{H})$ is of class $\mathcal{Q}$, i.e., $T$ has the block matrix form (1) with $V, E$ and $Q$ satisfying (2)-(5). What is more interesting, there may exist different orthogonal decompositions of $\mathcal{H}$ relative to which the given operator $T$ is of class $\mathcal{Q}$ and the Taylor spectra $\sigma(|Q|,|E|)$ of the corresponding pairs $(|Q|,|E|)$ are significantly different (see Example 7.3). It turns out that the class of Brownian isometries is the only subclass of $\mathcal{Q}$ considered in this paper which cannot be characterized by the Taylor spectrum $\sigma(|Q|,|E|)$ of the pair $(|Q|,|E|)$ (see Remark 8.5).

\section{Prerequisites}

In this section we fix notation and terminology and give necessary facts. Let $\mathbb{Z}, \mathbb{R}$ and $\mathbb{C}$ stand for the sets of integers, real numbers and complex numbers, respectively. Denote by $\mathbb{N}$ the set of positive integers. Set

$$
\begin{aligned}
& \mathbb{Z}_{+}=\{n \in \mathbb{Z}: n \geqslant 0\}, \quad \mathbb{R}_{+}=\{x \in \mathbb{R}: x \geqslant 0\}, \\
& \mathbb{D}_{+}=\left\{(s, t) \in \mathbb{R}_{+}^{2}: s^{2}+t^{2}<1\right\}, \quad \mathbb{T}_{+}=\left\{(s, t) \in \mathbb{R}_{+}^{2}: s^{2}+t^{2}=1\right\}, \\
& \overline{\mathbb{D}}_{+}=\mathbb{D}_{+} \cup \mathbb{T}_{+} .
\end{aligned}
$$

Given a set $X$, we write $\chi_{\Delta}$ for the characteristic function of a subset $\Delta$ of $X$. The $\sigma$-algebra of all Borel subsets of a topological space $X$ is denoted by $\mathfrak{B}(X)$. For $x \in X, \delta_{x}$ stands for the Borel probability measure on $\mathbb{R}$ supported on $\{x\}$.

Let $\mathcal{H}$ be a complex Hilbert space. We call an operator $T \in \boldsymbol{B}(\mathcal{H})$ a contraction (resp., an expansion) if $\|T h\| \leqslant\|h\|$ for all $h \in \mathcal{H}$ (resp., $\|T h\| \geqslant\|h\|$ for all $h \in \mathcal{H}$ ), or equivalently if $T^{*} T \leqslant I$ (resp., $T^{*} T \geqslant I$ ). The contractivity of $T$ can also be characterized by requiring that $\|T\| \leqslant 1$ (however $\|T\| \geqslant 1$ does not characterize expansivity of $T$ ). Obviously, $T$ is an isometry if and only $T$ is simultaneously a contraction and an expansion. We write $\sigma(T)$ for the spectrum of $T$. If $G$ is a regular Borel spectral measure on a topological Hausdorff space $X$, then supp $G$ denotes the closed support of $G$, i.e., $X \backslash \operatorname{supp} G$ is the largest open subset $\Delta$ of $X$ such that $G(\Delta)=0$. Recall that if $T \in \boldsymbol{B}(\mathcal{H})$ is a selfadjoint operator and $G$ is the spectral measure of $T$, then $\sigma(T)=\operatorname{supp} G$. The following elementary fact will be frequently used in this paper. 
Suppose that $T \in \boldsymbol{B}(\mathcal{H})$ is selfadjoint. If $a, b \in \mathbb{R}$ are such that $a \leqslant b$, then $\sigma(T) \subseteq[a, b]$ if and only if aI $\leqslant T \leqslant b I$. Moreover, if $T \geqslant 0$ and $0 \notin \sigma(T)$, then $\min \sigma(T)=\left\|T^{-1}\right\|^{-1}$ and $\max \sigma(T)=\|T\|$.

We refer the reader to [15, Chapter 6] for more details on spectral theory of Hilbert space operators.

A pair $\left(T_{1}, T_{2}\right)$ of commuting operators $T_{1}, T_{2} \in \boldsymbol{B}(\mathcal{H})$ is said to be a spherical contraction (resp., spherical expansion) if $T_{1}^{*} T_{1}+T_{2}^{*} T_{2} \leqslant I$ (resp., $\left.T_{1}^{*} T_{1}+T_{2}^{*} T_{2} \geqslant I\right)$. If $\left(T_{1}, T_{2}\right)$ is simultaneously spherical contraction and spherical expansion, that is $T_{1}^{*} T_{1}+T_{2}^{*} T_{2}=I$, then $\left(T_{1}, T_{2}\right)$ is called a spherical isometry (see [10]).

For a pair $\left(T_{1}, T_{2}\right)$ of commuting operators $T_{1}, T_{2} \in \boldsymbol{B}(\mathcal{H})$, we denote by $\sigma\left(T_{1}, T_{2}\right)$ the Taylor spectrum of $\left(T_{1}, T_{2}\right)$, and by $r\left(T_{1}, T_{2}\right)$ the geometric spectral radius of $\left(T_{1}, T_{2}\right)$, that is,

$$
r\left(T_{1}, T_{2}\right)=\max \left\{\left(\left|z_{1}\right|^{2}+\left|z_{2}\right|^{2}\right)^{1 / 2}:\left(z_{1}, z_{2}\right) \in \sigma\left(T_{1}, T_{2}\right)\right\} .
$$

The reader is referred to $[18,21,39,50,52]$ for the definitions and the basic properties of the Taylor spectrum and the geometric spectral radius (of commuting $n$-tuples of operators). In particular, the Taylor spectrum $\sigma\left(T_{1}, T_{2}\right)$ is a nonempty compact subset of $\mathbb{C}^{2}$ whenever $\mathcal{H} \neq\{0\}$. Moreover, it has the following projection property (see [50, Lemma 3.1]; see also [21, Theorem 4.9]):

$$
\pi_{j}\left(\sigma\left(T_{1}, T_{2}\right)\right)=\sigma\left(T_{j}\right), \quad j=1,2,
$$

where $\pi_{1}, \pi_{2}: \mathbb{C}^{2} \rightarrow \mathbb{C}$ are defined by $\pi_{1}\left(z_{1}, z_{2}\right)=z_{1}$ and $\pi_{2}\left(z_{1}, z_{2}\right)=z_{2}$ for $\left(z_{1}, z_{2}\right) \in \mathbb{C}^{2}$. The following fact follows directly from the projection property of the Taylor spectrum.

Suppose that $\mathcal{H} \neq\{0\}$ and $\lambda \in \mathbb{C}$. Then $\sigma\left(T_{1}, T_{2}\right) \subseteq\{\lambda\} \times \mathbb{C}$ if and only if $\sigma\left(T_{1}\right)=\{\lambda\}$. Moreover, if $\sigma\left(T_{1}\right)=\{\lambda\}$, then $\sigma\left(T_{1}, T_{2}\right)=\{\lambda\} \times \sigma\left(T_{2}\right)$.

The symmetric version with $\mathbb{C} \times\{\lambda\}$ in place of $\{\lambda\} \times \mathbb{C}$ holds as well.

Note that under the assumption of (8), $\sigma\left(T_{1}, T_{2}\right)=\sigma\left(T_{1}\right) \times \sigma\left(T_{2}\right)$ if $\sigma\left(T_{1}\right)=\{\lambda\}$ or if $\sigma\left(T_{2}\right)=\{\lambda\}$. However, the first equation may not hold even for positive operators (see (56) in Example 6.4).

For a given pair $\left(T_{1}, T_{2}\right)$ of commuting selfadjoint operators $T_{1}, T_{2} \in \boldsymbol{B}(\mathcal{H})$, there exists a unique Borel spectral measure $G: \mathfrak{B}\left(\mathbb{R}^{2}\right) \rightarrow \boldsymbol{B}(\mathcal{H})$, called the joint spectral measure of $\left(T_{1}, T_{2}\right)$, such that

$$
p\left(T_{1}, T_{2}\right)=\int_{\mathbb{R}^{2}} p\left(t_{1}, t_{2}\right) G\left(d t_{1}, d t_{2}\right), \quad p \in \mathbb{C}\left[x_{1}, x_{2}\right],
$$

where as usual $\mathbb{C}\left[x_{1}, x_{2}\right]$ stands for the ring of polynomials in indeterminates $x_{1}, x_{2}$ with complex coefficients (similar notations are used throughout the paper with no further explanation). The joint spectral measure $G$ is the product of the spectral measures of $T_{1}$ and $T_{2}$ (see [15, Theorem 6.5.1]). As shown below, in this particular case, the Taylor spectrum 
$\sigma\left(T_{1}, T_{2}\right)$ coincides with the closed support of the joint spectral measure $G$; this yields the spectral mapping theorem for continuous functions. ${ }^{3}$

Theorem 2.1 Suppose that $T_{1}, T_{2} \in \boldsymbol{B}(\mathcal{H})$ are commuting selfadjoint operators with the joint spectral measure $G$. Then the following assertions are valid:

(i) $\sigma\left(T_{1}, T_{2}\right)=\operatorname{supp} G$; moreover, if $T_{1}, T_{2}$ are positive, then $\sigma\left(T_{1}, T_{2}\right) \subseteq \mathbb{R}_{+}^{2}$,

(ii) for any continuous function $\psi: \sigma\left(T_{1}, T_{2}\right) \rightarrow \mathbb{R}$,

$$
\sigma\left(\psi\left(T_{1}, T_{2}\right)\right)=\psi\left(\sigma\left(T_{1}, T_{2}\right)\right),
$$

where $\psi\left(T_{1}, T_{2}\right):=\int_{\sigma\left(T_{1}, T_{2}\right)} \psi d G$,

(iii) for any continuous function $\psi=\left(\psi_{1}, \psi_{2}\right): \sigma\left(T_{1}, T_{2}\right) \rightarrow \mathbb{R}^{2}$,

$$
\sigma\left(\psi\left(T_{1}, T_{2}\right)\right)=\psi\left(\sigma\left(T_{1}, T_{2}\right)\right),
$$

where $\boldsymbol{\psi}\left(T_{1}, T_{2}\right):=\left(\psi_{1}\left(T_{1}, T_{2}\right), \psi_{2}\left(T_{1}, T_{2}\right)\right)$.

Proof First observe that by (7) we have

$$
\sigma\left(T_{1}, T_{2}\right) \subseteq \sigma\left(T_{1}\right) \times \sigma\left(T_{2}\right) \subseteq \mathbb{R}^{2},
$$

so if additionally $T_{1}$ and $T_{2}$ are positive, then $\sigma\left(T_{1}, T_{2}\right) \subseteq \mathbb{R}_{+}^{2}$.

(i) First note that the Taylor spectrum $\sigma\left(T_{1}, T_{2}\right)$ coincides with the left spectrum of $\left(T_{1}, T_{2}\right)$ (see [21, Proposition 7.2]). It is a routine matter to show that the left spectrum of $\left(T_{1}, T_{2}\right)$ coincides with the approximate point spectrum of $\left(T_{1}, T_{2}\right)$ (this is true for an arbitrary pair of commuting Hilbert space operators). Hence, for $\left(\lambda_{1}, \lambda_{2}\right) \in \mathbb{R}^{2}$, $\left(\lambda_{1}, \lambda_{2}\right) \notin \sigma\left(T_{1}, T_{2}\right)$ if and only if there exists $c \in(0, \infty)$ such that

$$
\left\|\left(T_{1}-\lambda_{1} I\right) h\right\|+\left\|\left(T_{2}-\lambda_{2} I\right) h\right\| \geqslant c\|h\|, \quad h \in \mathcal{H},
$$

or equivalently, by [15, Theorem 6.5.3], if and only if $\left(\lambda_{1}, \lambda_{2}\right) \notin \operatorname{supp} G$. Combined with (10), this proves (i).

(ii) Note that

$$
\sigma\left(\psi\left(T_{1}, T_{2}\right)\right)=\sigma\left(\int_{\sigma\left(T_{1}, T_{2}\right)} \psi d G\right) \stackrel{(*)}{=} \psi(\operatorname{supp} G) \stackrel{(\mathrm{i})}{=} \psi\left(\sigma\left(T_{1}, T_{2}\right)\right),
$$

where $(*)$ follows from [15, eq. (13), p. 158].

(iii) By [15, Theorem 6.6.4], Go $\psi_{j}^{-1}$ is the spectral measure of $\psi_{j}\left(T_{1}, T_{2}\right)$ for $j=1,2$. Let $\widetilde{G}$ be the product of these measures (see [15, Theorem 5.2.6]). Since

$$
\widetilde{G}\left(\Delta_{1} \times \Delta_{2}\right)=G\left(\psi_{1}^{-1}\left(\Delta_{1}\right)\right) G\left(\psi_{2}^{-1}\left(\Delta_{2}\right)\right)=G\left(\psi^{-1}\left(\Delta_{1} \times \Delta_{2}\right)\right), \quad \Delta_{1}, \Delta_{2} \in \mathfrak{B}(\mathbb{R}),
$$

we deduce from the uniqueness part of [15, Theorem 5.2.6] that $\widetilde{G}=G \circ \psi^{-1}$. Hence $G \circ \psi^{-1}$ is the joint spectral measure of the pair $\psi\left(T_{1}, T_{2}\right)$. This yields

\footnotetext{
${ }^{3}$ Note that Theorem 2.1 remains true for commuting normal operators with $\mathbb{C}$ in place of $\mathbb{R}$. We refer the reader to [51, Theorem 4.8] (see also [21, Theorem 5.19] and [39, Corollary IV.30.11]) for the spectral mapping theorem for the Taylor functional calculus.
} 


$$
\sigma\left(\boldsymbol{\psi}\left(T_{1}, T_{2}\right)\right) \stackrel{(\mathrm{i})}{=} \operatorname{supp} G \circ \boldsymbol{\psi}^{-1} \stackrel{(*)}{=} \boldsymbol{\psi}(\operatorname{supp} G) \stackrel{(\mathrm{i})}{=} \boldsymbol{\psi}\left(\sigma\left(T_{1}, T_{2}\right)\right) .
$$

(To get $(*)$ adapt the proof of [48, Lemma 3.2].) This completes the proof.

As a consequence of Theorem 2.1, we obtain the following.

If $T_{1}, T_{2} \in \boldsymbol{B}(\mathcal{H})$ are commuting and selfadjoint operators, then $\sigma\left(T_{1}, T_{2}\right) \subseteq(\mathbb{R} \times\{0\}) \cup(\{0\} \times \mathbb{R})$ if and only if $T_{1} T_{2}=0$, or equivalently

if $T_{1}=0 \oplus \widetilde{T}_{1}$ and $T_{2}=\widetilde{T}_{2} \oplus 0$ relative to $\mathcal{H}=\mathcal{M}\left(T_{1}\right) \oplus \overline{\mathscr{R}\left(T_{1}\right)}$.

For this, note that $\sigma\left(T_{1}, T_{2}\right) \subseteq(\mathbb{R} \times\{0\}) \cup(\{0\} \times \mathbb{R})$ if and only if $p\left(\sigma\left(T_{1}, T_{2}\right)\right)=0$, where $p(s, t)=s \cdot t$. Hence, applying Theorem 2.1(ii) gives the former equivalence in (11). The latter is a matter of routine verification.

The following lemma is surely folklore. For self-containedness we sketch its proof (the reader can easily formulate a version for commuting normal operators).

Lemma 2.2 Let $T_{1}, T_{2} \in \boldsymbol{B}(\mathcal{H})$ be commuting selfadjoint operators on a nonzero complex Hilbert space $\mathcal{H}$. Then

$$
r\left(T_{1}, T_{2}\right)=\left\|T_{1}^{2}+T_{2}^{2}\right\|^{1 / 2}=\min \left\{\delta \in \mathbb{R}_{+}: \sigma\left(T_{1}, T_{2}\right) \subseteq \delta \cdot \overline{\mathbb{D}}\right\},
$$

where $\mathbb{D}:=\left\{(s, t) \in \mathbb{R}^{2}: s^{2}+t^{2}<1\right\}$. Moreover, $T_{1}^{2}+T_{2}^{2}$ is invertible if and only if $\left\{\delta \in(0, \infty): \sigma\left(T_{1}, T_{2}\right) \subseteq \mathbb{R}^{2} \backslash \delta \cdot \mathbb{D}\right\} \neq \emptyset ;$ if this is the case, then

$$
\left\|\left(T_{1}^{2}+T_{2}^{2}\right)^{-1}\right\|^{-1 / 2}=\max \left\{\delta \in(0, \infty): \sigma\left(T_{1}, T_{2}\right) \subseteq \mathbb{R}^{2} \backslash \delta \cdot \mathbb{D}\right\} .
$$

Proof Since the proofs of (12) and (13) are similar, we justify only (13). Suppose $T_{1}^{2}+T_{2}^{2}$ is invertible. If $\delta \in(0, \infty)$ is such that $\sigma\left(T_{1}, T_{2}\right) \subseteq \mathbb{R}^{2} \backslash \delta \cdot \mathbb{D}$, then by Theorem 2.1(ii) with $\psi\left(x_{1}, x_{2}\right)=x_{1}^{2}+x_{2}^{2}$, we have

$$
\sigma\left(T_{1}^{2}+T_{2}^{2}\right)=\sigma\left(\psi\left(T_{1}, T_{2}\right)\right)=\psi\left(\sigma\left(T_{1}, T_{2}\right)\right) \subseteq\left[\delta^{2}, \infty\right),
$$

which implies that $T_{1}^{2}+T_{2}^{2}$ is invertible and

$$
\delta^{2} \leqslant \min \sigma\left(T_{1}^{2}+T_{2}^{2}\right) \stackrel{(6)}{=}\left\|\left(T_{1}^{2}+T_{2}^{2}\right)^{-1}\right\|^{-1} .
$$

Reversing the argument with $\delta=\left\|\left(T_{1}^{2}+T_{2}^{2}\right)^{-1}\right\|^{-1 / 2}$, we obtain the converse implication and (13). This completes the proof.

We now describe the Taylor spectrum of an orthogonal sum of pairs of commuting selfadjoint operators.

Proposition 2.3 Suppose that for every $n \in \mathbb{N},\left(T_{1, n}, T_{2, n}\right)$ is a pair of commuting selfadjoint operators on a nonzero complex Hilbert space $\mathcal{H}_{n}$. For $j=1,2$, let $T_{j}=\bigoplus_{n=1}^{\infty} T_{j, n}$. Then $\left(T_{1}, T_{2}\right)$ is a pair of commuting selfadjoint operators such that

$$
\sigma\left(T_{1}, T_{2}\right)=\overline{\bigcup_{n=1}^{\infty} \sigma\left(T_{1, n}, T_{2, n}\right)} .
$$


Proof Set $\boldsymbol{T}=\left(T_{1}, T_{2}\right)$ and $\boldsymbol{T}_{n}=\left(T_{1, n}, T_{2, n}\right)$ for $n \in \mathbb{N}$. Denote by $G_{T}$ and $G_{T_{n}}$ the joint spectral measures of $\boldsymbol{T}$ and $\boldsymbol{T}_{n}$, respectively. Let $G_{T_{j}}$ and $G_{T_{j, n}}$ be the spectral measures of $T_{j}$ and $T_{j, n}$, respectively, where $j=1,2$ and $n \in \mathbb{N}$. It is clear that

$$
G_{T_{j}}(\Delta)=\bigoplus_{n=1}^{\infty} G_{T_{j, n}}(\Delta), \quad \Delta \in \mathfrak{B}(\mathbb{R}), \quad j=1,2 .
$$

This implies that

$$
G_{T_{1}}\left(\Delta_{1}\right) G_{T_{2}}\left(\Delta_{2}\right)=\bigoplus_{n=1}^{\infty} G_{T_{n}}\left(\Delta_{1} \times \Delta_{2}\right), \quad \Delta_{1}, \Delta_{2} \in \mathfrak{B}(\mathbb{R}) .
$$

Combined with the uniqueness of joint spectral measures, this yields

$$
G_{T}(\Delta)=\bigoplus_{n=1}^{\infty} G_{T_{n}}(\Delta), \quad \Delta \in \mathfrak{B}\left(\mathbb{R}^{2}\right) .
$$

In view of Theorem 2.1(i), it suffices to show that

$$
\operatorname{supp} G_{T}=\overline{\bigcup_{n=1}^{\infty} \operatorname{supp} G_{T_{n}}}
$$

For this, take $(s, t) \in \mathbb{R}^{2}$. If $(s, t) \notin \operatorname{supp} G_{T}$, then there exists an open set $\Delta$ in $\mathbb{R}^{2}$ such that $(s, t) \in \Delta$ and $G_{T}(\Delta)=0$. Therefore by (15), $G_{T_{n}}(\Delta)=0$ for all $n \in \mathbb{N}$, which implies that $(s, t) \notin \operatorname{supp} G_{\boldsymbol{T}_{n}}$ for all $n \in \mathbb{N}$. As a consequence, $\bigcup_{n=1}^{\infty} \operatorname{supp} G_{\boldsymbol{T}_{n}} \subseteq \operatorname{supp} G_{\boldsymbol{T}}$, which shows that the right side of (16) is contained in the left side. In turn, if ${ }^{n}(s, t) \notin\left(\bigcup_{n=1}^{\infty} \operatorname{supp} G_{T_{n}}\right)^{-}$, then there exists an open set $\Delta$ in $\mathbb{R}^{2}$ such that $(s, t) \in \Delta$ and $\Delta \cap\left(\bigcup_{n=1}^{\infty} \operatorname{supp} G_{T_{n}}\right)^{-}=\emptyset$. Hence, $G_{T_{n}}(\Delta)=0$ for all $n \in \mathbb{N}$, which together with (15) implies that $G_{T}(\Delta)=0$. As a consequence, $(s, t) \notin \operatorname{supp} G_{T}$. This completes the proof.

Corollary 2.4 If $\Gamma$ is an arbitrary nonempty compact subset of $\mathbb{R}^{2}$ (resp. $\mathbb{R}_{+}^{2}$ ) and $\mathcal{H}$ is a separable infinite-dimensional complex Hilbert space, then there exists a pair $\left(T_{1}, T_{2}\right)$ of commuting selfadjoint (resp. positive) operators $T_{1}, T_{2} \in \boldsymbol{B}(\mathcal{H})$ such that $\Gamma=\sigma\left(T_{1}, T_{2}\right)$.

Proof Since $\mathbb{R}^{2}$ is separable metric space, so is $\Gamma$. Hence, there exists a sequence $\left\{\left(x_{1, n}, x_{2, n}\right)\right\}_{n=1}^{\infty} \subseteq \Gamma$ which is dense in $\Gamma$. The proof is completed by applying Proposition 2.3 to $\mathcal{H}_{n}=\mathbb{C}, T_{1, n}=x_{1, n} I_{\mathbb{C}}$ and $T_{2, n}=x_{2, n} I_{\mathbb{C}}$ and by observing that according to (8), $\sigma\left(T_{1, n}, T_{2, n}\right)=\left\{\left(x_{1, n}, x_{2, n}\right)\right\}$ for all $n \in \mathbb{N}$.

Remark 2.5 A closer inspection of the proof reveals that Proposition 2.3 remains valid for families (of arbitrary cardinality) of pairs of commuting normal operators. As a consequence, Corollary 2.4 remains true if selfadjoint operators are replaced by normal operators and $\mathbb{R}$ by $\mathbb{C}$. What is more, using only the definition of the Taylor spectrum, one can show that (14) holds (certainly without the closure) for any finite number of pairs of commuting operators (cf. [20]). 


\section{Fundamental properties of operators of class $\mathcal{Q}$}

In this section we prove some basic properties of operators of class $\mathcal{Q}$ that are needed in this paper. We begin by showing that the operators of class $\mathcal{Q}$ form a huge class which can be parameterized in a sense by arbitrary pairs of commuting positive operators.

Proposition 3.1 Let $\mathcal{H}=\mathcal{H}_{1} \oplus \mathcal{H}_{2}$ be a nontrivial orthogonal decomposition of a complex Hilbert space $\mathcal{H}$. Then the following assertions are valid:

(i) if $T=\left[\begin{array}{ll}V & E \\ 0 & Q\end{array}\right] \in \mathcal{Q}_{\mathcal{H}_{1}, \mathcal{H}_{2}}$, then $|Q|$ and $|E|$ are commuting positive operators such that $\operatorname{dim} \overline{\mathscr{R}(|E|)} \leqslant \operatorname{dim} \mathscr{R}(V)^{\perp}$,

(ii) if $V \in \boldsymbol{B}\left(\mathcal{H}_{1}\right)$ is an isometry and $A, B \in \boldsymbol{B}\left(\mathcal{H}_{2}\right)$ are commuting positive operators such that $\operatorname{dim} \overline{\mathscr{R}(B)} \leqslant \operatorname{dim} \mathscr{R}(V)^{\perp}$, then there exists $E \in \boldsymbol{B}\left(\mathcal{H}_{2}, \mathcal{H}_{1}\right)$ such that $T=\left[\begin{array}{cc}V & E \\ 0 & A\end{array}\right] \in \mathcal{Q}_{\mathcal{H}_{1}, \mathcal{H}_{2}}$ and $|E|=B$.

Moreover, if $T=\left[\begin{array}{ll}V & E \\ 0 & Q\end{array}\right] \in \mathcal{Q}_{\mathcal{H}_{1}, \mathcal{H}_{2}}$, then there exists $\widetilde{E} \in \boldsymbol{B}\left(\mathcal{H}_{2}, \mathcal{H}_{1}\right)$ such that $\left[\begin{array}{cc}V & \widetilde{E} \\ 0 & |Q|\end{array}\right] \in \mathcal{Q}_{\mathcal{H}_{1}, \mathcal{H}_{2}}$ and $|\widetilde{E}|=|E|$.

Proof (i) That $|Q|$ and $|E|$ commute follows from (4) and the square root theorem. Let $E=U|E|$ be the polar decomposition of $E$. Then $U$ maps $\overline{\mathscr{R}(|E|)}$ unitarily onto $\overline{\mathscr{R}(E)}$. Since by (3), $\overline{\mathscr{R}(E)} \subseteq \mathscr{R}(V)^{\perp}$, we are done.

(ii) Since $\operatorname{dim} \overline{\mathscr{R}(B)} \leqslant \operatorname{dim} \mathscr{R}(V)^{\perp}$, there exists a closed subspace $\mathcal{M}$ of $\mathscr{R}(V)^{\perp}$ such that $\operatorname{dim} \overline{\mathscr{R}(B)}=\operatorname{dim} \mathcal{M}$. Let $U \in \boldsymbol{B}\left(\mathcal{H}_{2}, \mathcal{H}_{1}\right)$ be a unique partial isometry with the initial space

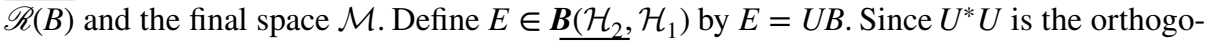
nal projection of $\mathcal{H}_{2}$ onto the initial space $\overline{\mathscr{R}(B)}$ of $U$, we get

$$
|E|^{2}=E^{*} E=B\left(U^{*} U\right) B=B^{2} .
$$

By the uniqueness of the square root, we deduce that $|E|=B$. It is easily seen that $T=\left[\begin{array}{ll}V & E \\ 0 & A\end{array}\right] \in \mathcal{Q}_{\mathcal{H}_{1}, \mathcal{H}_{2}}$.

The "moreover" part is a direct consequence of (i) and (ii). This completes the proof.

Corollary 3.2 Suppose that $\mathcal{H}_{2}$ is a nonzero complex Hilbert space and $A, B \in \boldsymbol{B}\left(\mathcal{H}_{2}\right)$ are commuting positive operators. Then there exist a nonzero complex Hilbert space $\mathcal{H}_{1}$, an isometry $V \in \boldsymbol{B}\left(\mathcal{H}_{1}\right)$ and an operator $E \in \boldsymbol{B}\left(\mathcal{H}_{2}, \mathcal{H}_{1}\right)$ such that $T=\left[\begin{array}{ll}V & E \\ 0 & A\end{array}\right] \in \mathcal{Q}_{\mathcal{H}_{1}, \mathcal{H}_{2}}$ (relative to $\left.\mathcal{H}=\mathcal{H}_{1} \oplus \mathcal{H}_{2}\right)$ and $|E|=B$.

Proof If $B=0$, then we can apply Proposition 3.1(ii) to any nonzero complex Hilbert space $\mathcal{H}_{1}$ and an arbitrary isometry $V \in \boldsymbol{B}\left(\mathcal{H}_{1}\right)$. In turn, if $B \neq 0$, then we can take an infinitedimensional complex Hilbert space $\mathcal{H}_{1}$ such that $\operatorname{dim} \overline{\mathscr{R}(B)} \leqslant \operatorname{dim} \mathcal{H}_{1}$. Then there exists an isometry $V \in \boldsymbol{B}\left(\mathcal{H}_{1}\right)$ such that

$$
\operatorname{dim} \overline{\mathscr{R}(B)} \leqslant \operatorname{dim} \mathcal{H}_{1}=\operatorname{dim} \mathscr{R}(V)^{\perp} .
$$

Applying Proposition 3.1(ii) completes the proof. 
The theorem below is crucial for our further investigations because the overwhelming majority of results of this paper are stated in terms of the Taylor spectrum of the pair $(|Q|,|E|)$.

Theorem 3.3 Suppose that $\Gamma$ is an arbitrary nonempty compact subset of $\mathbb{R}_{+}^{2}$ and $\mathcal{H}_{2}$ is a separable infinite-dimensional complex Hilbert space. Then there exists a nonzero complex Hilbert space $\mathcal{H}_{1}$ and $T=\left[\begin{array}{ll}V & E \\ 0 & Q\end{array}\right] \in \mathcal{Q}_{\mathcal{H}_{1}, \mathcal{H}_{2}}$ (relative to $\mathcal{H}=\mathcal{H}_{1} \oplus \mathcal{H}_{2}$ ) such that $\sigma(|Q|,|E|)=\Gamma$.

Proof It follows from Corollary 2.4 that there exists a pair $(A, B)$ of commuting positive operators $A, B \in \boldsymbol{B}\left(\mathcal{H}_{2}\right)$ such that $\sigma(A, B)=\Gamma$. Applying Corollary 3.2 completes the proof.

As shown below the norm of an operator of class $\mathcal{Q}$ can be expressed in terms of the geometric spectral radius of the pair $(|Q|,|E|)$.

Proposition 3.4 Suppose that $T=\left[\begin{array}{ll}V & E \\ 0 & Q\end{array}\right] \in \mathcal{Q}_{\mathcal{H}_{1}, \mathcal{H}_{2}}$. Then

$$
\|T\|=\max \{1, r(|Q|,|E|)\} .
$$

Proof Let $G$ be the joint spectral measure of the pair $(|Q|,|E|)$ and let $G$ - ess $\sup \varphi \operatorname{stand}$ for the essential supremum of a Borel function $\varphi: \mathbb{R}_{+}^{2} \rightarrow \mathbb{R}_{+}$with respect to the measure $G$. It follows from Definition 1.1 that

$$
T^{*} T=\left[\begin{array}{cc}
I & 0 \\
0 & Q^{*} Q+E^{*} E
\end{array}\right] .
$$

Combined with the hypothesis that the spaces $\mathcal{H}_{1}$ and $\mathcal{H}_{2}$ are nonzero, this implies that

$$
\begin{aligned}
\|T\|=\||T|\| & =\left\|\left[\begin{array}{ll}
I & 0 \\
0 & \left(|Q|^{2}+|E|^{2}\right)^{1 / 2}
\end{array}\right]\right\| \\
& =\max \left\{1,\left\|\left(|Q|^{2}+|E|^{2}\right)^{1 / 2}\right\|\right\} \\
& =\max \left\{1, G-\operatorname{ess~sup}_{(s, t) \in \mathbb{R}_{+}^{2}}\left(s^{2}+t^{2}\right)^{1 / 2}\right\} \\
& \stackrel{(*)}{=} \max \left\{1, \max _{(s, t) \in \sigma(|Q|,|E|)}\left(s^{2}+t^{2}\right)^{1 / 2}\right\}, \\
& =\max \{1, r(|Q|,|E|)\},
\end{aligned}
$$

where $(*)$ follows from Theorem 2.1(i) and the continuity of the function $(s, t) \mapsto\left(s^{2}+t^{2}\right)^{1 / 2}$ on $\mathbb{R}_{+}^{2}$. This completes the proof.

Remark 3.5 It follows from Proposition 3.4 that if $T \in \boldsymbol{B}(\mathcal{H})$ is of class $\mathcal{Q}$ and $\|T\|>1$, then the geometric spectral radius $r(|Q|,|E|)$ does not depend on the choice of an orthogonal decomposition $\mathcal{H}=\mathcal{H}_{1} \oplus \mathcal{H}_{2}$ of $\mathcal{H}$ relative to which $T$ has a block matrix representation (1) with entries $V, E$ and $Q$ satisfying the conditions (2)-(5). We refer the reader to Example 7.3 for a detailed discussion of the question of the existence of different orthogonal decompositions of the underlying Hilbert space $\mathcal{H}$ relative to which a given operator $T \in \boldsymbol{B}(\mathcal{H})$ is of class $\mathcal{Q}$. 
Next we characterize contractive, isometric and expansive operators of class $\mathcal{Q}$.

Proposition 3.6 Suppose $T=\left[\begin{array}{cc}V & E \\ 0 & Q\end{array}\right] \in \mathcal{Q}_{\mathcal{H}_{1}, \mathcal{H}_{2}}$. Then the following conditions are equivalent:

(i) T is a contraction (resp., an isometry, an expansion),

(ii) ( $|Q|,|E|)$ is a spherical contraction (resp., a spherical isometry, a spherical expansion),

(iii) $\sigma(|Q|,|E|) \subseteq \overline{\mathbb{D}}_{+}\left(\right.$resp., $\left.\sigma(|Q|,|E|) \subseteq \mathbb{T}_{+}, \sigma(|Q|,|E|) \subseteq \mathbb{R}_{+}^{2} \backslash \mathbb{D}_{+}\right)$.

Moreover, if $T$ is a contraction, then $\|T\|=1$.

Proof By Proposition 3.1(i), $(|Q|,|E|)$ is a pair of commuting positive operators. The equivalence (i) $\Leftrightarrow$ (ii) follows from (18). Next by applying Theorem 2.1(ii) to the polynomial $\psi\left(x_{1}, x_{2}\right)=x_{1}^{2}+x_{2}^{2}$, we get

$$
\psi(\sigma(|Q|,|E|))=\sigma\left(|Q|^{2}+|E|^{2}\right),
$$

which together with (6) and $\sigma(|Q|,|E|) \subseteq \mathbb{R}_{+}^{2}$ yields the equivalence (ii) $\Leftrightarrow$ (iii).

The "moreover" part is a direct consequence of Proposition 3.4.

For self-containedness, we state the following result whose straightforward proof is left to the reader.

Proposition 3.7 The class $\mathcal{Q}$ is closed under the operation of taking orthogonal sums, i.e., if $\left\{T_{l}\right\}_{l \in J}$ is a uniformly bounded family of operators of class $\mathcal{Q}$, then $\bigoplus_{l \in J} T_{l}$ is an operator of class $\mathcal{Q}$.

The following lemma provides a sufficient condition for the product of two quasinormal operators to be quasinormal.

Lemma 3.8 Suppose that $Q_{1}, Q_{2} \in \boldsymbol{B}(\mathcal{H})$ are commuting quasinormal operators such that $Q_{1}$ commutes with $Q_{2}^{*} Q_{2}$ and $Q_{2}$ commutes with $Q_{1}^{*} Q_{1}$. Then $Q_{1} Q_{2}$ is quasinormal. Moreover, any positive integer power of a quasinormal operator is quasinormal.

Proof We leave the simple algebraic proof of the first part to the reader. The "moreover" part follows from the first part by applying the formula

$$
Q^{* n} Q^{n}=\left(Q^{*} Q\right)^{n}, \quad n \in \mathbb{Z}_{+},
$$

which is valid for any quasinormal operator $Q$.

Our next goal is to give a sufficient condition for the product of two operators of class $\mathcal{Q}$ to be of class $\mathcal{Q}$.

Proposition 3.9 Suppose $T_{1}=\left[\begin{array}{cc}V_{1} & E_{1} \\ 0 & Q_{1}\end{array}\right] \in \mathcal{Q}_{\mathcal{H}_{1}, \mathcal{H}_{2}}$ and $T_{2}=\left[\begin{array}{cc}V_{2} & E_{2} \\ 0 & Q_{2}\end{array}\right] \in \mathcal{Q}_{\mathcal{H}_{1}, \mathcal{H}_{2}}$ are such that $Q_{k} Q_{l}^{*} Q_{l}=Q_{l}^{*} Q_{l} Q_{k}$ and $Q_{k} E_{l}^{*} E_{l}=E_{l}^{*} E_{l} Q_{k}$ for all distinct $k, l \in\{1,2\}$. 
Then $T_{1} T_{2}=\left[\begin{array}{ll}V & E \\ 0 & Q\end{array}\right] \in \mathcal{Q}_{\mathcal{H}_{1}, \mathcal{H}_{2}}$, where $V=V_{1} V_{2}, E=V_{1} E_{2}+E_{1} Q_{2}$ and $Q=Q_{1} Q_{2}$.

Proof First notice that

$$
T_{1} T_{2}=\left[\begin{array}{cc}
V_{1} V_{2} & V_{1} E_{2}+E_{1} Q_{2} \\
0 & Q_{1} Q_{2}
\end{array}\right] .
$$

Clearly, $V_{1} V_{2}$ is an isometry, while by Lemma 3.8, $Q_{1} Q_{2}$ is a quasinormal operator. Routine computations show that $\left(V_{1} V_{2}\right)^{*}\left(V_{1} E_{2}+E_{1} Q_{2}\right)=0$ and $Q_{1} Q_{2}$ commutes with $\left(V_{1} E_{2}+E_{1} Q_{2}\right)^{*}\left(V_{1} E_{2}+E_{1} Q_{2}\right)$ meaning that $T_{1} T_{2}$ is of class $\mathcal{Q}$.

It turns out that the operation of taking positive integer powers is inner in the class $\mathcal{Q}$. The class $\mathcal{Q}$ is also closed under the operation of taking the Cauchy dual. Furthermore, we discuss the questions of when an operator of class $\mathcal{Q}$ is $\Delta_{T}$-regular and when it satisfies the kernel condition introduced recently in [7].

Proposition 3.10 Suppose $T=\left[\begin{array}{ll}V & E \\ 0 & Q\end{array}\right] \in \mathcal{Q}_{\mathcal{H}_{1}, \mathcal{H}_{2}}$. Then

(i) $T^{n}=\left[\begin{array}{cc}V^{n} & E_{n} \\ 0 & Q^{n}\end{array}\right] \in \mathcal{Q}_{\mathcal{H}_{1}, \mathcal{H}_{2}} \quad$ for any $n \in \mathbb{Z}_{+}$, where

$$
E_{n}= \begin{cases}0 & \text { if } n=0, \\ \sum_{j=1}^{n} V^{j-1} E Q^{n-j} & \text { if } n \geqslant 1,\end{cases}
$$

(ii) $T^{* n} T^{n}=\left[\begin{array}{cc}I & 0 \\ 0 & \Omega_{n}\end{array}\right] \in \mathcal{Q}_{\mathcal{H}_{1}, \mathcal{H}_{2}} \quad$ for any $n \in \mathbb{Z}_{+}$, where

$$
\Omega_{n}= \begin{cases}I & \text { if } n=0, \\ E^{*} E\left(\sum_{j=0}^{n-1}\left(Q^{*} Q\right)^{j}\right)+\left(Q^{*} Q\right)^{n} & \text { if } n \geqslant 1,\end{cases}
$$

(iii) T is left-invertible if and only if $\Omega_{1}$ is invertible, or equivalently there exists $\delta \in(0, \infty)$ such that $\sigma(|Q|,|E|) \subseteq \mathbb{R}_{+}^{2} \backslash \delta \cdot \mathbb{D}_{+}$; if this is the case, then

$\max \left\{\delta \in \mathbb{R}_{+}: \sigma(|Q|,|E|) \subseteq \mathbb{R}_{+}^{2} \backslash \delta \cdot \mathbb{D}_{+}\right\}=\left\|\Omega_{1}^{-1}\right\|^{-1 / 2}$,

(iv) if $T$ is left-invertible, then $T^{\prime} \in \mathcal{Q}_{\mathcal{H}_{1}, \mathcal{H}_{2}}$ and

$$
T^{\prime}=\left[\begin{array}{ll}
V & E \Omega_{1}^{-1} \\
0 & Q \Omega_{1}^{-1}
\end{array}\right],
$$

(v) $T$ is $\Delta_{T}$-regular if and only if $T$ is an expansion,

(vi) $T$ satisfies the kernel condition, i.e., $T^{*} T \mathscr{N}\left(T^{*}\right) \subseteq \mathscr{N}\left(T^{*}\right)$, if and only if $\left(|Q|^{2}+|E|^{2}-I\right) E^{*} h_{1}=0$ for every $h_{1} \in \mathscr{N}\left(V^{*}\right)$ such that $E^{*} h_{1} \in \mathscr{R}\left(Q^{*}\right)$.

Proof (i) Using induction, one can verify that

$$
T^{n}=\left[\begin{array}{cc}
V^{n} & E_{n} \\
0 & Q^{n}
\end{array}\right], \quad n \in \mathbb{Z}_{+},
$$

where 


$$
E_{0}=0 \text { and } E_{n+1}=V E_{n}+E Q^{n} \text { for } n \in \mathbb{Z}_{+} .
$$

By induction, (24) implies (20). Clearly for any $n \in \mathbb{Z}_{+}, V^{n}$ is an isometry and, by Lemma 3.8, $Q^{n}$ is a quasinormal operator. Since $V$ is an isometry, we infer from (3) and (20) that $V^{* n} E_{n}=0$ for any $n \in \mathbb{Z}_{+}$. Employing (24), we see that

$$
\begin{aligned}
E_{n+1}^{*} E_{n+1} & \stackrel{(2) \&(3)}{=} E_{n}^{*} E_{n}+Q^{* n} E^{*} E Q^{n} \\
& \stackrel{(4) \&(19)}{=} E_{n}^{*} E_{n}+\left(Q^{*} Q\right)^{n} E^{*} E, \quad n \in \mathbb{Z}_{+} .
\end{aligned}
$$

Using induction and (4), we deduce that $Q$ commutes with $E_{n}^{*} E_{n}$ for all $n \in \mathbb{Z}_{+}$. This implies that $T^{n}$ is of class $\mathcal{Q}$ for any $n \in \mathbb{Z}_{+}$.

(ii) It follows from (i) and (19) that

$$
T^{* n} T^{n}=\left[\begin{array}{lc}
I & 0 \\
0 & E_{n}^{*} E_{n}+\left(Q^{*} Q\right)^{n}
\end{array}\right], \quad n \in \mathbb{Z}_{+} .
$$

Using induction, (25) and (4), we conclude that

$$
E_{n}^{*} E_{n}=E^{*} E \sum_{j=0}^{n-1}\left(Q^{*} Q\right)^{j}, \quad n \in \mathbb{N} .
$$

Combined with (26), this yields (ii).

(iii) It is clear that $T$ is left-invertible if and only if $T^{*} T$ is invertible, which by (ii) with $n=1$ is equivalent to the invertibility of $\Omega_{1}$. The remaining statement in (iii) is a direct consequence of Theorem 2.1(i) and Lemma 2.2.

(iv) It is a routine matter to show that (23) holds and then to verify that $T^{\prime}$ is of class $\mathcal{Q}$.

(v) The "only if" part is obvious. To prove the "if" part, notice that by (ii),

$$
\Delta_{T}=\left[\begin{array}{cc}
0 & 0 \\
0 & \Omega_{1}-I
\end{array}\right] .
$$

Since $T$ is an expansion, we see that $\Omega_{1}-I \geqslant 0$ and

$$
\Delta_{T}^{1 / 2}=\left[\begin{array}{cc}
0 & 0 \\
0 & \left(\Omega_{1}-I\right)^{1 / 2}
\end{array}\right] .
$$

Knowing that $Q$ commutes with $\Omega_{1}$ and using the square root theorem, we deduce that $Q$ commutes with $\left(\Omega_{1}-I\right)^{1 / 2}$, and consequently by (27), $\Delta_{T}^{1 / 2} T \Delta_{T}^{1 / 2}=\Delta_{T} T$, which means that $T$ is $\Delta_{T}$-regular.

(vi) Since $T^{*}=\left[\begin{array}{cc}V^{*} & 0 \\ E^{*} & Q^{*}\end{array}\right]$, we easily verify that

$$
\mathscr{N}\left(T^{*}\right)=\left\{h_{1} \oplus h_{2} \in \mathcal{H}: h_{1} \in \mathscr{N}\left(V^{*}\right) \text { and } E^{*} h_{1}+Q^{*} h_{2}=0\right\} .
$$

To prove the "if" part, suppose that

$$
\left(|Q|^{2}+|E|^{2}-I\right)\left(\left(E^{*} \mathscr{N}\left(V^{*}\right)\right) \cap \mathscr{R}\left(Q^{*}\right)\right)=\{0\} .
$$

If $h_{1} \oplus h_{2} \in \mathscr{N}\left(T^{*}\right)$, then in view of (28) and (29), we have 


$$
\begin{aligned}
E^{*} h_{1}+Q^{*}\left(|Q|^{2}+|E|^{2}\right) h_{2} \stackrel{(4) \&(5)}{=} E^{*} h_{1}+\left(|Q|^{2}+|E|^{2}\right) Q^{*} h_{2} \\
=\left(I-|Q|^{2}-|E|^{2}\right) E^{*} h_{1}=0 .
\end{aligned}
$$

Hence by (ii) with $n=1$ and (28), $T^{*} T\left(h_{1} \oplus h_{2}\right) \in \mathcal{N}\left(T^{*}\right)$, which justifies the "if" part. The "only if" part goes by reversing the above argument. This completes the proof.

Corollary 3.11 Suppose $T=\left[\begin{array}{cc}V & E \\ 0 & Q\end{array}\right] \in \mathcal{Q}_{\mathcal{H}_{1}, \mathcal{H}_{2}}$ satisfies the kernel condition, $E \neq 0$ and $\mathscr{R}\left(Q^{*}\right)=\mathcal{H}_{2}$. Then

(i) 1 is an eigenvalue of $|Q|^{2}+|E|^{2}$,

(ii) $\sigma(|Q|,|E|) \cap \mathbb{T}_{+} \neq \emptyset$.

Proof (i) Suppose, on the contrary, that 1 is not an eigenvalue of the operator $|Q|^{2}+|E|^{2}$. Then by Proposition 3.10(vi), $\mathscr{N}\left(V^{*}\right) \subseteq \mathscr{N}\left(E^{*}\right)$. This implies that $\mathscr{R}(E) \subseteq \mathscr{R}(V)$. Since by (3), $\mathscr{R}(E) \subseteq \mathscr{R}(V)^{\perp}$, we see that $E=0$, a contradiction.

(ii) By (i) and Theorem 2.1(ii) applied to the polynomial $\psi\left(x_{1}, x_{2}\right)=x_{1}^{2}+x_{2}^{2}$, we have $1 \in \sigma(\psi(|Q|,|E|))=\psi(\sigma(|Q|,|E|))$, so there exists $(s, t) \in \sigma(|Q|,|E|) \subseteq \mathbb{R}_{+}^{2}$ such that $\psi(s, t)=1$, which completes the proof.

\section{Moment theoretic necessities}

In this section we prove a series of lemmata concerning Hamburger and Stieltjes moment problems needed in subsequent sections of this paper. We state some of them in a more general context, namely for the multi-dimensional moment problems, because the proofs are essentially the same.

Below we use the standard multi-index notation, that is, if $d \in \mathbb{N}$, $\alpha=\left(\alpha_{1}, \ldots, \alpha_{d}\right) \in \mathbb{Z}_{+}^{d}$ and $x=\left(x_{1}, \ldots, x_{d}\right) \in \mathbb{R}^{d}$, then we write $x^{\alpha}=x_{1}^{\alpha_{1}} \cdots x_{d}^{\alpha_{d}}$. A complex Borel measure $\mu$ on $\mathbb{R}^{d}$ is said to be compactly supported if there is a compact subset $K$ of $\mathbb{R}^{d}$ such that $|\mu|\left(\mathbb{R}^{d} \backslash K\right)=0$, where $|\mu|$ denotes the total variation measure of $\mu$. We write $\operatorname{supp} \mu$ for the closed support of a finite positive Borel measure $\mu$ on $\mathbb{R}^{d}$ (the support exists because such $\mu$ is automatically regular, see [43, Theorem 2.18]). We say that a multi-sequence $\left\{\gamma_{\alpha}\right\}_{\alpha \in \mathbb{Z}^{d}} \subseteq \mathbb{R}$ is a Hamburger moment multi-sequence (or Hamburger moment sequence if $d=1$ ) if there exists a positive Borel measure $\mu$ on $\mathbb{R}^{d}$, called a representing measure of $\left\{\gamma_{\alpha}\right\}_{\alpha \in \mathbb{Z}_{+}^{d}}$, such that

$$
\gamma_{\alpha}=\int_{\mathbb{R}^{d}} x^{\alpha} d \mu(x), \quad \alpha \in \mathbb{Z}_{+}^{d} .
$$

If such $\mu$ is unique, then $\left\{\gamma_{\alpha}\right\}_{\alpha \in \mathbb{Z}_{+}^{d}}$ is said to be determinate. If (30) holds for some positive Borel measure $\mu$ on $\mathbb{R}^{d}$ supported in $\mathbb{R}_{+}^{d}$, then $\left\{\gamma_{\alpha}\right\}_{\alpha \in \mathbb{Z}_{+}^{d}}$ is called a Stieltjes moment multisequence (or Stieltjes moment sequence if $d=1$ ).

Lemma 4.1 Let $d \in \mathbb{N}$. Suppose that $\mu_{1}$ and $\mu_{2}$ are compactly supported complex Borel measures on $\mathbb{R}^{d}$ such that 


$$
\int_{\mathbb{R}^{d}} x^{\alpha} d \mu_{1}(x)=\int_{\mathbb{R}^{d}} x^{\alpha} d \mu_{2}(x), \quad \alpha \in \mathbb{Z}_{+}^{d} .
$$

Then $\mu_{1}=\mu_{2}$.

Proof Since $\left|\mu_{1}-\mu_{2}\right|(\Delta) \leqslant\left|\mu_{1}\right|(\Delta)+\left|\mu_{2}\right|(\Delta)$ for all Borel subsets $\Delta$ of $\mathbb{R}^{d}$, the complex Borel measure $\mu:=\mu_{1}-\mu_{2}$ is compactly supported, that is supp $|\mu| \subseteq[-R, R]^{d}$ for some $R \in \mathbb{R}_{+}$, and

$$
\int_{\mathbb{R}^{d}} p d \mu=0, \quad p \in \mathbb{C}\left[x_{1}, \ldots, x_{d}\right]
$$

Let $f$ be a continuous complex function on $\mathbb{R}^{d}$ vanishing at infinity. By the Stone-Weierstrass theorem, there exists a sequence $\left\{p_{n}\right\}_{n=1}^{\infty} \subseteq \mathbb{C}\left[x_{1}, \ldots, x_{d}\right]$ such that

$$
\lim _{n \rightarrow \infty} \sup _{x \in[-R, R]^{d}}\left|f(x)-p_{n}(x)\right|=0 .
$$

Since

$$
\begin{aligned}
\left|\int_{\mathbb{R}^{d}} f d \mu\right| & \stackrel{(31)}{=}\left|\int_{\mathbb{R}^{d}}\left(f-p_{n}\right) d \mu\right| \leqslant \int_{[-R, R]^{d}}\left|f-p_{n}\right| d|\mu| \\
& \leqslant|\mu|\left([-R, R]^{d}\right) \sup _{x \in[-R, R]^{d}}\left|f(x)-p_{n}(x)\right|, \quad n \in \mathbb{N},
\end{aligned}
$$

we deduce from (32) that $\int_{\mathbb{R}^{d}} f d \mu=0$. Applying [43, Theorems 6.19 and 2.18] yields $\mu=0$, or equivalently, $\mu_{1}=\mu_{2}$.

Remark 4.2 Concerning Lemma 4.1, it is worth mentioning that any sequence $\left\{\gamma_{n}\right\}_{n=0}^{\infty} \subseteq \mathbb{C}$ has infinitely many representing complex measures. For this, note that there exists a complex Borel measure $\rho$ on $\mathbb{R}$ such that (see $[16,25,41]$ )

$$
\gamma_{n}=\int_{\mathbb{R}} x^{n} d \rho(x), \quad n \in \mathbb{Z}_{+} .
$$

Let $\left\{s_{n}\right\}_{n=0}^{\infty}$ be an indeterminate Hamburger moment sequence with two distinct representing measures $\mu_{1}$ and $\mu_{2}$ (see $\left.[13,46]\right)$. Then $\mu:=\mu_{1}-\mu_{2}$ is a signed Borel measure on $\mathbb{R}$ such that

$$
\int_{\mathbb{R}} x^{n} d \mu(x)=0, \quad n \in \mathbb{Z}_{+}
$$

As a consequence, we have

$$
\gamma_{n}=\int_{\mathbb{R}} x^{n} d(\rho+\vartheta \mu)(x), \quad n \in \mathbb{Z}_{+}, \vartheta \in \mathbb{C} .
$$

Moreover, the mapping $\mathbb{C} \ni \vartheta \longmapsto \rho+\vartheta \mu$ is an injection.

Lemma 4.3 If $d \in \mathbb{N}, R=\left(R_{1}, \ldots, R_{d}\right) \in \mathbb{R}_{+}^{d}$ and $\mu$ is a complex Borel measure on $\mathbb{R}^{d}$ such that supp $|\mu| \subseteq\left[-R_{1}, R_{1}\right] \times \cdots \times\left[-R_{d}, R_{d}\right]$, then 


$$
\left|\int_{\mathbb{R}^{d}} x^{\alpha} d \mu(x)\right| \leqslant|\mu|\left(\mathbb{R}^{d}\right) R^{\alpha}, \quad \alpha \in \mathbb{Z}_{+}^{d} .
$$

Proof Since $\left|x^{\alpha}\right| \leqslant R^{\alpha}$ for all $\alpha \in \mathbb{Z}_{+}^{d}$ and $x \in \operatorname{supp}|\mu|$, we get

$$
\left|\int_{\mathbb{R}^{d}} x^{\alpha} d \mu(x)\right| \leqslant \int_{\mathbb{R}^{d}}\left|x^{\alpha}\right| d|\mu|(x) \leqslant|\mu|\left(\mathbb{R}^{d}\right) R^{\alpha}, \quad \alpha \in \mathbb{Z}_{+}^{d} .
$$

Lemma 4.4 Let $d \in \mathbb{N}, \mu$ be a compactly supported complex Borel measure on $\mathbb{R}^{d}$ and $\gamma_{\alpha}=\int_{\mathbb{R}^{d}} x^{\alpha} d \mu(x)$ for $\alpha \in \mathbb{Z}_{+}^{d}$. Then the following conditions are equivalent:

(i) $\left\{\gamma_{\alpha}\right\}_{\alpha \in \mathbb{R}^{d}}$ is a Hamburger moment multi-sequence,

(ii) $\mu$ is a positive measure.

Moreover, if (i) holds, then $\left\{\gamma_{\alpha}\right\}_{\alpha \in \mathbb{R}^{d}}$ is determinate.

Proof (i) $\Rightarrow$ (ii) Let $v$ be a representing measure of $\left\{\gamma_{\alpha}\right\}_{\alpha \in \mathbb{Z}_{+}^{d}}$. By Lemma 4.3,

$$
\lim _{n \rightarrow \infty}\left(\int_{\mathbb{R}^{d}} x_{j}^{2 n} d v(x)\right)^{1 / 2 n}=\lim _{n \rightarrow \infty}\left|\int_{\mathbb{R}^{d}} x_{j}^{2 n} d \mu(x)\right|^{1 / 2 n} \leqslant R_{j}, \quad j=1, \ldots, d,
$$

where $R_{1}, \ldots, R_{d}$ are as in Lemma 4.3. Thus, by [43, Exercise 4(e), p. 71] (see also [45, Problem 1(a), p. 332]), supp $v \subseteq\left[-R_{1}, R_{1}\right] \times \ldots \times\left[-R_{d}, R_{d}\right]$. Hence by Lemma 4.1, $\left\{\gamma_{\alpha}\right\}_{\alpha \in \mathbb{R}^{d}}$ is determinate, $\mu=v$ and so $\mu$ is a positive measure.

The implication (ii) $\Rightarrow$ (i) is trivial.

We state now the following fact which we need in the proof of Lemma 4.6. It can be proved by induction on the degree of the polynomial in question.

Lemma 4.5 ([23, Exercise 7.2]) If $p \in \mathbb{C}[x]$ is of degree $k \in \mathbb{Z}_{+}$, then

$$
\left(\Delta^{m} \check{p}\right)_{n}=p^{(m)}(0), \quad n \in \mathbb{Z}_{+}, m \geqslant k,
$$

where $\Delta: \mathbb{C}^{\mathbb{Z}_{+}} \rightarrow \mathbb{C}^{\mathbb{Z}_{+}}$is the linear transformation given by $(\Delta \gamma)_{n}=\gamma_{n+1}-\gamma_{n}$ for $n \in \mathbb{Z}_{+}$ and $\gamma \in \mathbb{C}^{\mathbb{Z}_{+}}, \check{p} \in \mathbb{C}^{\mathbb{Z}_{+}}$is given by $\check{p}_{n}=p(n)$ for $n \in \mathbb{Z}_{+}$and $p^{(m)}(0)$ stands for the mth derivative of $p$ at 0 .

As shown below, a nonconstant polynomial perturbation of a Hamburger moment sequence is never a Hamburger moment sequence.

Lemma 4.6 Let $\left\{\gamma_{n}\right\}_{n=0}^{\infty}$ be a Hamburger moment sequence having a compactly supported representing measure $\mu$ and let $p \in \mathbb{R}[x]$. Then the following conditions are equivalent:

(i) the sequence $\left\{\gamma_{n}+p(n)\right\}_{n=0}^{\infty}$ is a Hamburger moment sequence,

(ii) $p$ is a constant polynomial and $\mu(\{1\})+p(0) \geqslant 0$. 
Moreover, if (ii) holds, then $\mu+p(0) \delta_{1}$ is a compactly supported representing measure of $\left\{\gamma_{n}+p(n)\right\}_{n=0}^{\infty}$.

Proof Without loss of generality we may assume that the polynomial $p$ is nonzero, that is $k:=\operatorname{deg} p \geqslant 0$.

(i) $\Rightarrow$ (ii) Define $\left\{\tilde{\gamma}_{n}\right\}_{n=0}^{\infty}$ by

$$
\tilde{\gamma}_{n}=\gamma_{n}+p(n), \quad n \in \mathbb{Z}_{+} .
$$

Let $\tilde{\mu}$ be a representing measure of $\left\{\tilde{\gamma}_{n}\right\}_{n=0}^{\infty}$. Applying Lemma 4.3 to $\left\{\gamma_{n}\right\}_{n=0}^{\infty}$ and using the fact that $\sup _{n \in \mathbb{Z}_{+}}|p(n)| e^{-n}<\infty$, we deduce that the measure $\tilde{\mu}$ is compactly supported (see the proof of Lemma 4.4). Since, by Lemma $4.5,\left(\Delta^{k} \check{p}\right)_{n}=p^{(k)}(0)$ for all $n \in \mathbb{Z}_{+}$, applying $\Delta^{k}$ to both sides of (33) yields

$$
\int_{\mathbb{R}} x^{n}(x-1)^{k} d \tilde{\mu}(x)=\int_{\mathbb{R}} x^{n}(x-1)^{k} d \mu(x)+p^{(k)}(0), \quad n \in \mathbb{Z}_{+} .
$$

Together with Lemma 4.1, this implies that

$$
\int_{\Delta}(x-1)^{k} d \tilde{\mu}(x)=\int_{\Delta}(x-1)^{k} d \mu(x)+p^{(k)}(0) \delta_{1}(\Delta), \quad \Delta \in \mathfrak{B}(\mathbb{R}) .
$$

If $k \geqslant 1$, then by substituting $\Delta=\{1\}$, we get $p^{(k)}(0)=0$, which gives a contradiction. Therefore, $p$ must be a constant polynomial. Substituting $k=0$ into (34), we get (ii).

The implication (ii) $\Rightarrow$ (i) and the "moreover" part are obvious.

The following is an immediate consequence of Lemma 4.6 applied to $\gamma_{n}=0$ and $\mu=0$.

Lemma 4.7 For $p \in \mathbb{R}[x]$, the following conditions are equivalent:

(i) $\{p(n)\}_{n=0}^{\infty}$ is a Hamburger moment sequence,

(ii) $\{p(n)\}_{n=0}^{\infty}$ is a Stieltjes moment sequence,

(iii) $\quad p$ is a constant polynomial and $p(0) \geqslant 0$.

Remark 4.8 The implication (i) $\Rightarrow$ (iii) of Lemma 4.7 can be proved more directly. Let $\mu$ be a representing measure of $\{p(n)\}_{n=0}^{\infty}$. Clearly, $p(0)=\mu(\mathbb{R}) \geqslant 0$. Suppose, on the contrary, that $k:=\operatorname{deg} p \geqslant 1$. By the Schwarz inequality, we have

$$
p(n)^{2}=\left(\int_{\mathbb{R}} x^{0} x^{n} d \mu(x)\right)^{2} \leqslant \int_{\mathbb{R}} x^{0} d \mu(x) \int_{\mathbb{R}} x^{2 n} d \mu(x)=p(0) p(2 n), \quad n \in \mathbb{Z}_{+} .
$$

Denote by $a$ the leading coefficient of $p$. The above inequality implies that

$$
a^{2}=\lim _{n \rightarrow \infty} \frac{p(n)^{2}}{n^{2 k}} \leqslant \lim _{n \rightarrow \infty} \frac{p(0) p(2 n)}{n^{2 k}}=0,
$$

which contradicts the fact that $a \neq 0$. Therefore, $p$ is a constant polynomial.

For the sake of completeness, we provide a proof of the following lemma which will be used in subsequent parts of this paper. 
Lemma 4.9 Let $G: \mathfrak{B}(X) \rightarrow \boldsymbol{B}(\mathcal{H})$ be a regular spectral measure on a topological Hausdorff space $X$ with compact support, $\varphi: X \rightarrow \mathbb{C}$ be a continuous function and $\Sigma$ be a relatively open subset of $\operatorname{supp} G$. Then the spectral integral $\int_{\Sigma} \varphi d G$, which is a bounded operator, is positive if and only if $\Sigma \subseteq\{x \in X: \varphi(x) \geqslant 0\}$.

Proof Since $\sup _{x \in \operatorname{supp} G}|\varphi(x)|<\infty, \int_{\Sigma} \varphi d G \in \boldsymbol{B}(\mathcal{H})$. To prove the "only if" part, assume that $\int_{\Sigma} \varphi d G \geqslant 0$. Then $\int_{\Sigma} \varphi(x)\langle G(d x) h, h\rangle \geqslant 0$ for all $h \in \mathcal{H}$. Substituting $G(\Delta) h$ in place of $h$ with $\Delta \in \mathfrak{B}(\Sigma)$, we see that $\int_{\Delta} \varphi(x)\langle G(d x) h, h\rangle \geqslant 0$ for all $\Delta \in \mathfrak{B}(\Sigma)$ and $h \in \mathcal{H}$. Combined with [9, Theorem 1.6.11], this implies that $\left\langle G\left(K_{\varphi}\right) h, h\right\rangle=0$ for all $h \in \mathcal{H}$, where $K_{\varphi}:=\left\{x \in \Sigma: \varphi(x) \in \mathbb{C} \backslash \mathbb{R}_{+}\right\}$. Since $K_{\varphi}$ is a relatively open subset of supp $G$ and $G\left(K_{\varphi}\right)=0$, we conclude that $K_{\varphi}=\emptyset$, which means that $\Sigma \subseteq\{x \in X: \varphi(x) \geqslant 0\}$. The "if" part is obvious.

Lemma 4.10 Let $G: \mathfrak{B}(X) \rightarrow \boldsymbol{B}(\mathcal{H})$ be a regular spectral measure on a topological Hausdorff space $X$ with compact support and let $\varphi_{n}: X \rightarrow \mathbb{R}, n \in \mathbb{Z}_{+}$, be continuous functions. Then the following conditions are equivalent:

(i) $\left\{\varphi_{n}(x)\right\}_{n=0}^{\infty}$ is a Stieltjes moment sequence for every $x \in \operatorname{supp} G$,

(ii) $\left\{\int_{X} \varphi_{n}(x)\langle G(d x) h, h\rangle\right\}_{n=0}^{\infty}$ is a Stieltjes moment sequence for every $h \in \mathcal{H}$.

Proof As in Lemma 4.9, $\int_{X} \varphi d G \in \boldsymbol{B}(\mathcal{H})$ whenever $\varphi: X \rightarrow \mathbb{C}$ is continuous.

(i) $\Rightarrow$ (ii) This can be easily deduced from [14, Theorem 6.2.5] (see also [7, Lemma 3.2]).

(ii) $\Rightarrow$ (i) Fix $n \in \mathbb{Z}_{+}$and $\lambda=\left(\lambda_{0}, \ldots, \lambda_{n}\right) \in \mathbb{C}^{n+1}$. Define the continuous function $\Phi_{\lambda}: X \rightarrow \mathbb{C}$ by

$$
\Phi_{\lambda}(x)=\sum_{k, l=0}^{n} \varphi_{k+l}(x) \lambda_{k} \bar{\lambda}_{l}, \quad x \in X .
$$

Applying the implication (iii) $\Rightarrow$ (i) of [14, Theorem 6.2.5], we see that

$$
\int_{X} \Phi_{\lambda}(x)\langle G(d x) h, h\rangle=\sum_{k, l=0}^{n} \int_{X} \varphi_{k+l}(x)\langle G(d x) h, h\rangle \lambda_{k} \bar{\lambda}_{l} \geqslant 0, \quad h \in \mathcal{H} .
$$

Hence $\int_{X} \Phi_{\lambda} d G \geqslant 0$, so by Lemma $4.9, \Phi_{\lambda}(x) \geqslant 0$ for all $x \in \operatorname{supp} G$, that is

$$
\sum_{k, l=0}^{n} \varphi_{k+l}(x) \lambda_{k} \bar{\lambda}_{l} \geqslant 0, \quad x \in \operatorname{supp} G .
$$

A similar argument shows that

$$
\sum_{k, l=0}^{n} \varphi_{k+l+1}(x) \lambda_{k} \bar{\lambda}_{l} \geqslant 0, \quad x \in \operatorname{supp} G .
$$

Finally, by applying the implication (i) $\Rightarrow$ (iii) of [14, Theorem 6.2.5], we complete the proof. 
Before concluding this section, we recall the celebrated criterion for subnormality of bounded operators essentially due to Lambert (see [37]; see also [47, Proposition 2.3]).

An operator $T \in \boldsymbol{B}(\mathcal{H})$ is subnormal if and only if for every $h \in \mathcal{H}$,

$\left\{\left\|T^{n} h\right\|^{2}\right\}_{n=0}^{\infty}$ is a Stieltjes moment sequence.

The following general characterization of subnormal operators fits nicely into the scope of the present investigations. It will be used to provide the second proof of Corollary 5.2.

Theorem 4.11 Suppose that $\varphi_{n}: X \rightarrow \mathbb{R}, n \in \mathbb{Z}_{+}$, are continuous functions on a topological Hausdorff space $X$ of the form

$$
\varphi_{n}(x)=\int_{\mathbb{R}_{+}} t^{n} d \mu_{x}(t), \quad n \in \mathbb{Z}_{+}, x \in X,
$$

where each $\mu_{x}$ is a compactly supported complex Borel measure on $\mathbb{R}_{+}$. Furthermore, assume that $T \in \boldsymbol{B}(\mathcal{H})$ is an operator for which there exists a regular spectral measure $G: \mathfrak{B}(X) \rightarrow \boldsymbol{B}(\mathcal{H})$ with compact support such that

$$
T^{* n} T^{n}=\int_{X} \varphi_{n}(x) G(d x), \quad n \in \mathbb{Z}_{+} \cdot
$$

Then $T$ is subnormal if and only if $\mu_{x}$ is a positive measure for every $x \in \operatorname{supp} G$.

Proof By (35) and (37), the operator $T$ is subnormal if and only if the sequence $\left\{\int_{X} \varphi_{n}(x)\langle G(d x) h, h\rangle\right\}_{n=0}^{\infty}$ is a Stieltjes moment sequence for every $h \in \mathcal{H}$. By Lemma 4.10, the latter holds if and only if $\left\{\varphi_{n}(x)\right\}_{n=0}^{\infty}$ is a Stieltjes moment sequence for every $x \in \operatorname{supp} G$, which in view of (36) and Lemma 4.4 is equivalent to the fact that $\mu_{x}$ is a positive measure for every $x \in \operatorname{supp} G$.

\section{Proof of the main result and some consequences}

Before proving Theorem 1.2, which is the main result of this paper, we make the following useful observation being a direct consequence of (8) and (10).

$$
\text { If } T=\left[\begin{array}{ll}
V & E \\
0 & Q
\end{array}\right] \in \mathcal{Q}_{\mathcal{H}_{1}, \mathcal{H}_{2}} \text {, then } E \neq 0 \text { if and only if } \sigma_{\sharp}(|Q|,|E|) \neq \emptyset \text {, }
$$

where $\sigma_{\sharp}(|Q|,|E|)=\sigma(|Q|,|E|) \cap\left(\mathbb{R}_{+} \times(0, \infty)\right)$.

Proof of Theorem 1.2 (i) $\Leftrightarrow$ (iii) In view of Proposition 3.1(i), $(|Q|,|E|)$ is a pair of commuting positive operators. Let $G$ be the joint spectral measure of $(|Q|,|E|)$. Then, by Theorem 2.1(i) and [43, Theorem 2.18], the measure $G$ is compactly supported and regular. It follows from (9) and (21) that

$$
\Omega_{n}=\int_{\mathbb{R}_{+}^{2}} \varphi_{n} d G, \quad n \in \mathbb{Z}_{+},
$$

where $\varphi_{n}: \mathbb{R}_{+}^{2} \rightarrow \mathbb{R}_{+}$is the continuous function defined by 


$$
\varphi_{n}(s, t)=\left\{\begin{array}{ll}
1 & \text { if } n=0, \\
t^{2}\left(\sum_{j=0}^{n-1} s^{2 j}\right)+s^{2 n} & \text { if } n \geqslant 1,
\end{array} \quad(s, t) \in \mathbb{R}_{+}^{2} .\right.
$$

Notice that by Proposition 3.10(ii) and (35), the operator $T$ is subnormal if and only if $\left\{\left\langle\Omega_{n} h, h\right\rangle\right\}_{n=0}^{\infty}$ is a Stieltjes moment sequence for every $h \in \mathcal{H}_{2}$. Hence in view of (39) and Lemma 4.10, $T$ is subnormal if and only if supp $G \subseteq \Xi$, where $\Xi$ is the set of all points $(s, t) \in \mathbb{R}_{+}^{2}$ for which $\left\{\varphi_{n}(s, t)\right\}_{n=0}^{\infty}$ is a Stieltjes moment sequence. Therefore, according to Theorem 2.1(i), to get the equivalence (i) $\Leftrightarrow$ (iii), it is enough to show that $\Xi=\overline{\mathbb{D}}_{+} \cup\left(\mathbb{R}_{+} \times\{0\}\right)$. For this purpose, take $(s, t) \in \mathbb{R}_{+}^{2}$ and consider two cases.

CASE $1 s=1$.

Then by (40), we have $\varphi_{n}(s, t)=1+n t^{2}$. Applying Lemma 4.7 to $p(x)=1+t^{2} x$, we see that $(1, t) \in \Xi$ if and only if $t=0$.

CASE $2 s \neq 1$.

Then by (40) we have

$$
\varphi_{n}(s, t)=\frac{t^{2}}{1-s^{2}}+\left(1-\frac{t^{2}}{1-s^{2}}\right) s^{2 n}, \quad n \in \mathbb{Z}_{+} .
$$

This implies that

$$
\varphi_{n}(s, t)=\int_{\mathbb{R}_{+}} x^{n} \mu_{s, t}(d x), \quad n \in \mathbb{Z}_{+},
$$

where $\mu_{s, t}: \mathfrak{B}\left(\mathbb{R}_{+}\right) \rightarrow \mathbb{R}$ is the signed measure of the form

$$
\mu_{s, t}=\frac{t^{2}}{1-s^{2}} \delta_{1}+\left(1-\frac{t^{2}}{1-s^{2}}\right) \delta_{s^{2}} .
$$

Using Lemma 4.4, we conclude that $(s, t) \in \Xi$ if and only if the measure $\mu_{s, t}$ is positive, or equivalently if and only if

$$
0 \leqslant \frac{t^{2}}{1-s^{2}} \leqslant 1
$$

If $t=0$, then (44) holds. If $t \neq 0$, then (44) holds if and only if $(s, t) \in \overline{\mathbb{D}}_{+}$. Thus $(s, t) \in \Xi$ if and only if $(s, t) \in\left(\overline{\mathbb{D}}_{+} \cup\left(\mathbb{R}_{+} \times\{0\}\right)\right) \backslash\{(1,0)\}$.

Summarizing Cases 1 and 2 , we conclude that $\Xi=\overline{\mathbb{D}}_{+} \cup\left(\mathbb{R}_{+} \times\{0\}\right)$, which gives the desired equivalence (i) $\Leftrightarrow$ (iii).

(ii) $\Leftrightarrow$ (iii) This is obvious due to the fact that $\sigma(|Q|,|E|) \subseteq \mathbb{R}_{+}^{2}$ (see Theorem 2.1(i)).

Before proving the equivalence (ii) $\Leftrightarrow$ (iv), we make necessary preparations. Set $K=\sigma(|Q|,|E|)$. Let $G_{|Q|}$ and $G_{|E|}$ be the spectral measures of $|Q|$ and $|E|$, respectively. Since $P$ is the orthogonal projection of $\mathcal{H}_{2}$ onto $\left.\mathcal{H}_{2} \ominus \mathscr{N}|E|\right)$ and $\left.\mathscr{N}|E|\right)=\mathscr{R}\left(G_{|E|}(\{0\})\right)$, we see that

$$
P=G_{|E|}((0, \infty))
$$

By Proposition 3.1(i), $|Q|$ commutes with $|E|$ so it commutes with $G_{|E|}$. As a consequence, the operators $|Q|,|E|$ and $P$ commute. Combined with Theorem 2.1(i), this yields 


$$
\begin{aligned}
\int_{\sigma_{\sharp}(|Q|,|E|)}\left(s^{2}+t^{2}\right) G(d s, d t) & =\int_{K \cap\left(\mathbb{R}_{+} \times(0, \infty)\right)}\left(s^{2}+t^{2}\right) G(d s, d t) \\
& =\int_{\mathbb{R}_{+} \times(0, \infty)}\left(s^{2}+t^{2}\right) G(d s, d t) \\
& =\int_{\mathbb{R}_{+}} s^{2} G_{|Q|}(d s) G_{|E|}((0, \infty))+\int_{(0, \infty)} t^{2} G_{|E|}(d t) \\
& =|Q|^{2} G_{|E|}((0, \infty))+|E|^{2} . \\
& =(|Q| P)^{2}+|E|^{2} .
\end{aligned}
$$

(ii) $\Rightarrow$ (iv) Suppose that (ii) holds. Then by (46), we have

$$
(|Q| P)^{2}+|E|^{2}=\int_{\sigma_{\sharp}(|Q|,|E|)}\left(s^{2}+t^{2}\right) G(d s, d t) \leqslant G\left(\sigma_{\sharp}(|Q|,|E|)\right) \leqslant I,
$$

which means that $(|Q| P,|E|)$ is a spherical contraction.

(iv) $\Rightarrow$ (ii) Suppose now that (iv) holds, i.e., $(|Q| P)^{2}+|E|^{2} \leqslant I$. Since $I_{\mathcal{H}_{2}}-P$ is the orthogonal projection of $\mathcal{H}_{2}$ onto $\mathscr{N}|E|$ ), we deduce that

$$
(|Q| P)^{2}+|E|^{2} \leqslant P .
$$

Observe now that

$$
\begin{aligned}
G\left(\sigma_{\sharp}(|Q|,|E|)\right) & =G\left(K \cap\left(\mathbb{R}_{+} \times(0, \infty)\right)\right) \\
& =G\left(\mathbb{R}_{+} \times(0, \infty)\right)=G_{|E|}((0, \infty)) \stackrel{(45)}{=} P .
\end{aligned}
$$

Combined with (46) and (47), this leads to

$$
\int_{\left.\sigma_{\sharp}|Q|,|E|\right)}\left(1-\left(s^{2}+t^{2}\right)\right) G(d s, d t) \geqslant 0 .
$$

Since $\sigma_{\sharp}(|Q|,|E|)$ is a relatively open subset of $\sigma(|Q|,|E|)$, we infer from Theorem 2.1(i) and Lemma 4.9 that $\sigma_{\sharp}(|Q|,|E|) \subseteq \overline{\mathbb{D}}_{+}$.

(iv) $\Leftrightarrow$ (v) That $\mathscr{M}=\mathscr{\mathscr { R }}(|E|)$ reduces $|Q|$ and $|E|$ follows from the fact that $P$ commutes

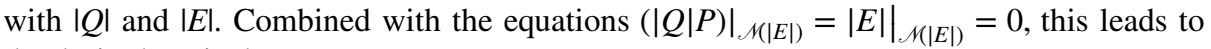
the desired equivalence.

(v) $\Leftrightarrow$ (vi) This equivalence can be proved in the same way as the equivalence (ii) $\Leftrightarrow$ (iii) of Proposition 3.6.

The "moreover" part is a direct consequence of (iii) and (10). This completes the proof.

In the rest of this section we record some consequences of Theorem 1.2. We begin with the following corollary which is immediate from Theorem 1.2(v).

Corollary 5.1 Suppose that $T=\left[\begin{array}{ll}V & E \\ 0 & Q\end{array}\right] \in \mathcal{Q}_{\mathcal{H}_{1}, \mathcal{H}_{2}}$ and $z=\left(z_{1}, z_{2}, z_{3}\right) \in \mathbb{C}^{3}$ is such that $\left|z_{1}\right|=1$ and $\left|z_{j}\right| \leqslant 1$ for $j=2,3$. Then $T_{z}:=\left[\begin{array}{cc}z_{1} V & z_{2} E \\ 0 & z_{3} Q\end{array}\right] \in \mathcal{Q}_{\mathcal{H}_{1}, \mathcal{H}_{2}}$. Moreover, if $T$ is subnormal, then so is $T_{z}$. 
The next corollary follows from Proposition 3.6 and Theorem 1.2 (recall that by Proposition 3.4 the contractions of class $\mathcal{Q}$ are of norm 1).

Corollary 5.2 Any contraction of class $\mathcal{Q}$ is subnormal.

As shown below, Corollary 5.2 can also be deduced from Theorem 4.11.

Second Proof of Corollary 5.2 Assume that $T$ is a contraction. Let $\widetilde{G}$ be the joint spectral measure of $(|Q|,|E|)$. Set $X=\overline{\mathbb{D}}_{+}$. It follows from Proposition 3.6 that $\sigma(|Q|,|E|) \subseteq X$. Hence, by Theorem 2.1(i), the function $G: \mathfrak{B}(X) \rightarrow \boldsymbol{B}(\mathcal{H})$ defined by

$$
G(\Delta)=\delta_{(1,0)}(\Delta) I_{\mathcal{H}_{1}} \oplus \widetilde{G}(\Delta), \quad \Delta \in \mathfrak{B}(X),
$$

is a spectral measure. In view of Proposition 3.10(ii) and (39), the condition (37) holds with $\varphi_{n}$ as in (40). Moreover, by (42) and (43), the condition (36) holds, where $\mu_{x}$ is the positive Borel measure on $\mathbb{R}_{+}$given by (43) for $x=(s, t) \in X \backslash\{(1,0)\}$ and $\mu_{(1,0)}=\delta_{1}$. Hence, by Theorem 4.11, $T$ is subnormal.

Below we indicate two subclasses of $\mathcal{Q}$ for which subnormality is completely characterized by contractivity.

Corollary 5.3 Suppose that $T=\left[\begin{array}{ll}V & E \\ 0 & Q\end{array}\right] \in \mathcal{Q}_{\mathcal{H}_{1}, \mathcal{H}_{2}}$, where $E=\alpha U, \alpha \in \mathbb{C} \backslash\{0\}$ and $U \in \boldsymbol{B}\left(\mathcal{H}_{2}, \mathcal{H}_{1}\right)$ is an isometry. Then the following conditions are equivalent:

(i) Tis subnormal,

(ii) $\|Q\|^{2}+|\alpha|^{2} \leqslant 1$,

(iii) $T$ is a contraction.

Proof By (8), we have

$$
\sigma(|Q|,|E|)=\sigma\left(|Q|,|\alpha| I_{\mathcal{H}_{2}}\right)=\sigma(|Q|) \times\{|\alpha|\} .
$$

Since

$$
\max \sigma(|Q|)=\||Q|\|=\|Q\|,
$$

we deduce from (48) that

$$
\sigma(|Q|,|E|) \subseteq \overline{\mathbb{D}}_{+} \text {if and only if }\|Q\|^{2}+|\alpha|^{2} \leqslant 1 .
$$

(i) $\Leftrightarrow$ (ii) Using the assumption that $\alpha \neq 0$ and applying Theorem 1.2, we deduce from (48) and (50) that $T$ is subnormal if and only if $\|Q\|^{2}+|\alpha|^{2} \leqslant 1$.

(ii) $\Leftrightarrow$ (iii) This is a direct consequence of (50) and Proposition 3.6.

The following is a variant of Corollary 5.3 with essentially the same proof.

Corollary 5.4 Suppose $T=\left[\begin{array}{ll}V & E \\ 0 & Q\end{array}\right] \in \mathcal{Q}_{\mathcal{H}_{1}, \mathcal{H}_{2}}$, where $Q=\alpha U, \alpha \in \mathbb{C}$ and $U \in \boldsymbol{B}\left(\mathcal{H}_{2}\right)$ is an isometry. If $E \neq 0$, then the following conditions are equivalent: 
(i) $T$ is subnormal,

(ii) $|\alpha|^{2}+\|E\|^{2} \leqslant 1$,

(iii) $T$ is a contraction.

\section{A solution to the Cauchy dual subnormality problem in the class $\mathcal{Q}$}

We begin by providing a complete answer to the question of when the Cauchy dual of an operator of class $\mathcal{Q}$ is subnormal.

Theorem 6.1 Suppose that $T=\left[\begin{array}{ll}V & E \\ 0 & Q\end{array}\right] \in \mathcal{Q}_{\mathcal{H}_{1}, \mathcal{H}_{2}}$ is left-invertible. Then $T^{\prime}$ is subnormal if and only if $\sigma(|Q|,|E|) \subseteq\left(\mathbb{R}_{+}^{2} \backslash \mathbb{D}_{+}\right) \cup\left(\mathbb{R}_{+} \times\{0\}\right)$.

Proof Since $T$ is left invertible, we infer from Proposition 3.10(iii) that $\Omega_{1}$ is invertible and

$$
\sigma(|Q|,|E|) \subseteq\left\{(s, t) \in \mathbb{R}_{+}^{2}: s^{2}+t^{2} \geqslant\left\|\Omega_{1}^{-1}\right\|^{-1}\right\} .
$$

Therefore, the function $\psi: \sigma(|Q|,|E|) \rightarrow \mathbb{R}^{2}$ given by

$$
\boldsymbol{\psi}(s, t)=\left(\frac{s}{s^{2}+t^{2}}, \frac{t}{s^{2}+t^{2}}\right), \quad(s, t) \in \sigma(|Q|,|E|),
$$

is well defined and continuous. By Proposition 3.10(iv), $T^{\prime} \in \mathcal{Q}_{\mathcal{H}_{1}, \mathcal{H}_{2}}$ and

$$
T^{\prime}=\left[\begin{array}{cc}
V & \tilde{E} \\
0 & \tilde{Q}
\end{array}\right],
$$

where $\tilde{E}:=E \Omega_{1}^{-1}$ and $\tilde{Q}:=Q \Omega_{1}^{-1}$. It is easily seen that

$$
|\tilde{Q}|=|Q|\left(|Q|^{2}+|E|^{2}\right)^{-1} \text { and }|\tilde{E}|=|E|\left(|Q|^{2}+|E|^{2}\right)^{-1} .
$$

Using the Stone-von Neumann functional calculus and Theorem 2.1(iii), we obtain

$$
\sigma(|\tilde{Q}|,|\tilde{E}|)=\sigma(\boldsymbol{\psi}(|Q|,|E|))=\psi(\sigma(|Q|,|E|)) .
$$

Applying Theorem 1.2(iii) to $T^{\prime}$ in place of $T$ and using (51), (52) and (53), we complete the proof.

We now show that within the class $\mathcal{Q}$ the Cauchy dual subnormality problem has an affirmative solution. What is more surprising is that we can solve it affirmatively even if complete hyperexpansivity is replaced by expansivity. For a more detailed discussion of this question, see Proposition 9.6 and Example 9.7. The solution is given in Corollary 6.2 below which is a direct consequence of Proposition 3.6 and Theorem 6.1. Another way of obtaining Corollary 6.2 is to apply Proposition 3.10(iv), Corollary 5.2 and the well-known and easy to prove fact that the Cauchy dual of an expansive operator is a contraction.

Corollary 6.2 The Cauchy dual of an expansive operator of class $\mathcal{Q}$ is a subnormal contraction. 
Below we recapture the affirmative solution to the Cauchy dual subnormality problem for quasi-Brownian isometries.

Corollary 6.3 ([7, Theorem 4.5]) The Cauchy dual of a quasi-Brownian isometry is a subnormal contraction.

Proof Let $T \in \boldsymbol{B}(\mathcal{H})$ be a quasi-Brownian isometry. If $T$ is an isometry, then $T^{\prime}=T$ is subnormal. If $T$ is not an isometry, then by [38, Proposition 5.1], $T$ has the block matrix form (1) with entries satisfying the conditions (2), (3) and (4), $Q$ being an isometry. Since each isometry is quasinormal, we deduce that $T$ is an operator of class $\mathcal{Q}$ and $Q^{*} Q+E^{*} E \geqslant I$. Combined with Proposition 3.6 and Corollary 6.2, this implies that $T^{\prime}$ is a subnormal contraction, which completes the proof.

Regarding Corollaries 5.2 and 6.2, it is worth pointing out that there are subnormal operators of class $\mathcal{Q}$ that are not contractive, and nonexpansive left-invertible operators of class $\mathcal{Q}$ whose Cauchy dual operators are subnormal. This can be deduced from Theorems 1.2(iii) and 6.1 and Propositions 3.6 and 3.10(iii) via an abstract nonexplicit procedure given in Theorem 3.3. Explicit instances are given in Example 6.4 below which will be continued in Sects. 9 and 10 under different circumstances.

Example 6.4 Our goal in this example is to show that

$1^{\circ}$ for any $\theta \in(1, \infty)$, there exists a subnormal operator $T$ of class $\mathcal{Q}$ such that $\|T\|=\theta$,

$2^{\circ}$ for any $\vartheta \in(0,1)$, there exists $T=\left[\begin{array}{ll}V & E \\ 0 & Q\end{array}\right] \in \mathcal{Q}_{\mathcal{H}_{1}, \mathcal{H}_{2}}$ such that $T$ is left-invertible, $T^{\prime}$ is subnormal and $\left\|\Omega_{1}^{-1}\right\|^{-1}=\vartheta(c f$. (21) and (22)).

For this purpose, let $\mathcal{K}$ be an infinite-dimensional complex Hilbert space and $\tau, \eta$ be complex numbers such that $\eta \neq 0$. Take a nonunitary isometry $V \in \boldsymbol{B}(\mathcal{K})$ and a quasinormal operator $\tilde{Q} \in \boldsymbol{B}(\mathscr{R}(V))$. Define the operators $Q_{\tau}, E_{\eta} \in \boldsymbol{B}(\mathcal{K})$ by

$$
Q_{\tau}=\tau I_{\left.\mathscr{N} V^{*}\right)} \oplus \tilde{Q} \text { and } E_{\eta}=\eta P,
$$

where $P \in \boldsymbol{B}(\mathcal{K})$ is the orthogonal projection of $\mathcal{K}$ onto $\mathscr{N}\left(V^{*}\right)$. Then the operator $Q_{\tau}$ is quasinormal. It is easily seen that $T_{\tau, \eta}:=\left[\begin{array}{ll}V & E_{\eta} \\ 0 & Q_{\tau}\end{array}\right] \in \mathcal{Q}_{\mathcal{K}}$ (see Definition 1.1). The operators $\left|Q_{\tau}\right|$ and $\left|E_{\eta}\right|$ can be represented relative to the orthogonal decomposition $\mathcal{K}=\mathscr{N}\left(V^{*}\right) \oplus \mathscr{R}(V)$ as follows:

$$
\left|Q_{\tau}\right|=|\tau| I_{\mathscr{N}\left(V^{*}\right)} \oplus|\tilde{Q}|, \quad\left|E_{\eta}\right|=|\eta| I_{\mathscr{N}\left(V^{*}\right)} \oplus 0 .
$$

Since $\mathscr{N}\left(V^{*}\right) \neq\{0\}$, we infer from (8) and Remark 2.5 that

$$
\begin{aligned}
\sigma\left(\left|Q_{\tau}\right|,\left|E_{\eta}\right|\right) & =\sigma\left(|\tau| I_{\mathscr{N}\left(V^{*}\right)},|\eta| I_{\mathscr{N}\left(V^{*}\right)}\right) \cup \sigma(|\tilde{Q}|, 0) \\
& =\{(|\tau|,|\eta|)\} \cup(\sigma(|\tilde{Q}|) \times\{0\}) .
\end{aligned}
$$

According to (6), (54) and (55), the following chain of equivalences holds 


$$
\sigma\left(\left|Q_{\tau}\right|,\left|E_{\eta}\right|\right)=\sigma\left(\left|Q_{\tau}\right|\right) \times \sigma\left(\left|E_{\eta}\right|\right) \Longleftrightarrow \sigma\left(\left|Q_{\tau}\right|\right)=\{|\tau|\} \Longleftrightarrow\left|Q_{\tau}\right|=|\tau| I .
$$

Combined with (55), Theorem 1.2(ii) implies that

$$
T_{\tau, \eta} \text { is subnormal if and only if }(|\tau|,|\eta|) \in \overline{\mathbb{D}}_{+} .
$$

Since by (54),

$$
E_{\eta}^{*} E_{\eta}+Q_{\tau}^{*} Q_{\tau}=\left(|\tau|^{2}+|\eta|^{2}\right) I_{\mathscr{N}\left(V^{*}\right)} \oplus|\tilde{Q}|^{2},
$$

we deduce from Proposition 3.10(iii) that

$$
T_{\tau, \eta} \text { is left-invertible if and only if }|\tilde{Q}| \text { is invertible. }
$$

In turn, Theorem 6.1 and (55) together yield the following:

$$
\begin{aligned}
& \text { if } T_{\tau, \eta} \text { is left-invertible, then } T_{\tau, \eta}^{\prime} \text { is subnormal if and only if } \\
& (|\tau|,|\eta|) \in \mathbb{R}_{+}^{2} \backslash \mathbb{D}_{+} \text {. }
\end{aligned}
$$

It follows from Proposition 3.4 and (55) that (cf. (49))

$$
\left\|T_{\tau, \eta}\right\|=\max \left\{1, \sqrt{|\tau|^{2}+|\eta|^{2}},\|\tilde{Q}\|\right\} .
$$

We are now ready to justify $1^{\circ}$ and $2^{\circ}$. If $\theta \in(1, \infty),(|\tau|,|\eta|) \in \overline{\mathbb{D}}_{+}$and $\tilde{Q}$ is chosen so that $\|\tilde{Q}\|=\theta$, then in view of (57) and (61), $T_{\tau, \eta}$ is a subnormal operator of class $\mathcal{Q}$ such that $\left\|T_{\tau, \eta}\right\|=\theta$, which proves $1^{\circ}$. In turn, if $\vartheta \in(0,1),(|\tau|,|\eta|) \in \mathbb{R}_{+}^{2} \backslash \mathbb{D}_{+}$and $\tilde{Q}$ is chosen to be invertible with ${ }^{4}\left\||\tilde{Q}|^{-1}\right\|^{2}=\vartheta^{-1}$, then in view of (58), (59) and (60), $T_{\tau, \eta}$ is a left-invertible operator of class $\mathcal{Q}$ such that $T_{\tau, \eta}^{\prime}$ is subnormal and $\left\|\left(E_{\eta}^{*} E_{\eta}+Q_{\tau}^{*} Q_{\tau}\right)^{-1}\right\|^{-1}=\vartheta$, which yields $2^{\circ}$.

\section{Quasi-Brownian isometries of class $\mathcal{Q}$}

In this section we provide a few characterizations of quasi-Brownian isometries of class $\mathcal{Q}$. Given an isometry $V \in \boldsymbol{B}(\mathcal{H})$, we say that $\mathcal{H}=\mathcal{H}_{1} \oplus \mathcal{H}_{2}$ is the von Neumann-Wold decomposition of $\mathcal{H}$ for $V$ if $\mathcal{H}_{1}=\bigcap_{n=0}^{\infty} V^{n}(\mathcal{H})$ and $\mathcal{H}_{2}=\bigoplus_{n=0}^{\infty} V^{n} \mathscr{N}\left(V^{*}\right)$; recall that $\mathcal{H}_{1}$ reduces $V$ to a unitary operator and $\mathcal{H}_{2}$ reduces $V$ to a unilateral shift of multiplicity $\operatorname{dim} \mathscr{N} V^{*}$ ) (see [49, Theorem I.1.1] for more details). It is clear that

$$
\left.\mathcal{H}_{2}=\bigoplus_{n=0}^{\infty} V^{n} \mathscr{N}\left(\left.V\right|_{\mathcal{H}_{2}}\right)^{*}\right)
$$

Theorem 7.1 Suppose $T=\left[\begin{array}{cc}V & E \\ 0 & Q\end{array}\right] \in \mathcal{Q}_{\mathcal{H}_{1}, \mathcal{H}_{2}}$. Then the following conditions are equivalent:

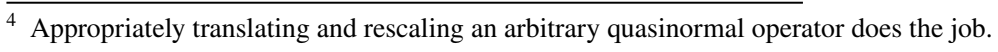


(i) T is a quasi-Brownian isometry,

(ii) $T$ is a 2-isometry,

(iii) $\left(|Q|^{2}-I\right)\left(|Q|^{2}+|E|^{2}-I\right)=0$,

(iv) $\sigma(|Q|,|E|) \subseteq \mathbb{T}_{+} \cup\left(\{1\} \times \mathbb{R}_{+}\right)$,

(v) there exists an orthogonal decomposition $\mathcal{H}_{2}=\mathcal{H}_{\mathrm{i}} \oplus \mathcal{H}_{\mathrm{si}}$ (zero summands are allowed) such that

(a) $\mathcal{H}_{\mathrm{i}}$ and $\mathcal{H}_{\mathrm{si}}$ reduce both $Q$ and $|E|$,

(b) $\left.Q\right|_{\mathcal{H}_{\mathrm{i}}}$ is an isometry and $\left(\left.Q\right|_{\mathcal{H}_{\mathrm{si}}},|E|_{\mathcal{H}_{\mathrm{si}}}\right)$ is a spherical isometry.

Moreover, if $\mathcal{H}_{\mathrm{i}}$ and $\mathcal{H}_{\mathrm{si}}$ are as in $(\mathrm{v})$ and $\mathcal{H}_{\mathrm{i}}=\mathcal{H}_{\mathrm{u}} \oplus \mathcal{H}_{\mathrm{s}}$ is the von Neumann-Wold decomposition of $\mathcal{H}_{\mathrm{i}}$ for $\left.Q\right|_{\mathcal{H}_{\mathrm{i}}}$, then $\mathcal{H}_{\mathrm{u}}$ and $\mathcal{H}_{\mathrm{s}}$ reduce both $Q$ and $|E|,\left.Q\right|_{\mathcal{H}_{\mathrm{u}}}$ is a unitary operator, and $\left.Q\right|_{\mathcal{H}_{\mathrm{s}}}$ is a unilateral shift (of finite or infinite multiplicity).

Proof (i) $\Leftrightarrow$ (ii) If $T$ is 2 -isometric, then by [42, Lemma 1], $T^{*} T \geqslant I$. This together with Proposition 3.10(v) shows that (i) and (ii) are equivalent.

(ii) $\Leftrightarrow$ (iii) This equivalence is a straightforward consequence of (4) and Proposition 3.10(ii).

(iii) $\Leftrightarrow$ (iv) Apply Theorem 2.1(ii) to $\psi\left(x_{1}, x_{2}\right)=\left(x_{1}^{2}-1\right)\left(x_{1}^{2}+x_{2}^{2}-1\right)$ and use (6).

(iii) $\Rightarrow$ (v) Since $Q$ is quasinormal, $|Q|^{2}-I$ commutes with $Q$ and so $\mathcal{H}_{\mathrm{i}}:=\mathscr{M}\left(|Q|^{2}-I\right)$ reduces $Q$ to an isometry. Set $\mathcal{H}_{\mathrm{si}}=\mathcal{H}_{2} \ominus \mathcal{H}_{\mathrm{i}}=\overline{\mathscr{R}\left(|Q|^{2}-I\right)}$. Clearly, $\mathcal{H}_{2}=\mathcal{H}_{\mathrm{i}} \oplus \mathcal{H}_{\mathrm{si}}$ and $\mathcal{H}_{\text {si }}$ reduces $Q$. Since $|Q|^{2}-I$ commutes with $|E|$, we see that $\mathcal{H}_{\mathrm{i}}$, and consequently $\mathcal{H}_{\mathrm{si}}$, reduces $|E|$. Notice that

$$
\left(|Q|^{2}+|E|^{2}\right)\left(|Q|^{2}-I\right)=\left(|Q|^{2}-I\right)\left(|Q|^{2}+|E|^{2}\right) \stackrel{(\text { iii) }}{=}|Q|^{2}-I,
$$

which implies that $|Q|^{2}+|E|^{2}$ is the identity operator on $\mathcal{H}_{\mathrm{si}}$. This shows that $\left(\left.Q\right|_{\mathcal{H}_{\mathrm{si}}},|E|_{\mathcal{H}_{\mathrm{si}}}\right)$ is a spherical isometry.

(v) $\Rightarrow$ (iii) This implication is a matter of routine verification.

We now prove the "moreover" part. Let $\mathcal{H}_{\mathrm{i}}=\mathcal{H}_{\mathrm{u}} \oplus \mathcal{H}_{\mathrm{s}}$ be the von Neumann-Wold decomposition of $\mathcal{H}_{\mathrm{i}}$ for $\left.Q\right|_{\mathcal{H}_{\mathrm{i}}}$. Since $\mathcal{H}_{\mathrm{u}}$ and $\mathcal{H}_{\mathrm{s}}$ reduce $\left.Q\right|_{\mathcal{H}_{\mathrm{i}}}$ and $\mathcal{H}_{\mathrm{i}}$ reduces $Q$, we deduce that $\mathcal{H}_{\mathrm{u}}$ and $\mathcal{H}_{\mathrm{s}}$ reduce $Q$, the operator $\left.Q\right|_{\mathcal{H}_{\mathrm{u}}}$ is unitary and the operator $\left.Q\right|_{\mathcal{H}_{\mathrm{s}}}$ is a unilateral shift (of finite or infinite multiplicity). Because $\mathcal{H}_{\mathrm{u}}=\bigcap_{n=0}^{\infty} Q^{n}\left(\mathcal{H}_{\mathrm{i}}\right),|E|\left(\mathcal{H}_{\mathrm{i}}\right) \subseteq \mathcal{H}_{\mathrm{i}}$ and $Q$ commutes with $|E|$, we see that

$$
|E|\left(\mathcal{H}_{\mathrm{u}}\right) \subseteq \bigcap_{n=0}^{\infty} Q^{n}|E|\left(\mathcal{H}_{\mathrm{i}}\right) \subseteq \mathcal{H}_{\mathrm{u}},
$$

which implies that $\mathcal{H}_{\mathrm{u}}$ reduces $|E|$. Since $\mathcal{H}_{\mathrm{si}}$ also reduces $|E|$, we conclude that $\mathcal{H}_{\mathrm{s}}$ reduces $|E|$. This completes the proof.

Below we show that there are operators of class $\mathcal{Q}$ with injective $E$, which are not 2-isometries (the case when $E=0$ is obvious due to the fact that quasinormal 2-isometries are isometric; see [27, Theorem 1 in \$2.6.2] and [32, Theorem 3.4]).

Corollary 7.2 Suppose $T=\left[\begin{array}{ll}V & E \\ 0 & Q\end{array}\right] \in \mathcal{Q}_{\mathcal{H}_{1}, \mathcal{H}_{2}}$, where $E$ is an isometry. Then the following conditions are equivalent:

(i) $T$ is a 2-isometry,

(ii) $Q=0 \oplus U$, where $U \in \boldsymbol{B}\left(\mathcal{H}_{2} \ominus \mathscr{N}(Q)\right)$ is an isometry. 
Proof In view of the equivalence of (ii) and (iii) in Theorem 7.1, $T$ is a 2-isometry if and only if $\left(Q^{*} Q\right)^{2}=Q^{*} Q$. Hence, by [30, Problem 127], $T$ is a 2-isometry if and only if $Q$ is a partial isometry. Since $Q$ is quasinormal, we infer from [30, Problem 204] that $Q$ is a partial isometry if and only if $Q=0 \oplus U$, where $U \in \boldsymbol{B}\left(\mathcal{H}_{2} \ominus \mathscr{N}(Q)\right)$ is an isometry.

Taking any quasinormal operator $Q$ which is not of the form as in the condition (ii) of Corollary 7.2 (e.g., when $\|Q\| \notin\{0,1\}$ ), we get an operator of class $\mathcal{Q}$ which is not a 2-isometry.

The key role which plays the Taylor spectrum $\sigma(|Q|,|E|)$ in the present paper raises the question of the existence of different orthogonal decompositions of the underlying Hilbert space $\mathcal{H}$ relative to which a given operator $T \in \boldsymbol{B}(\mathcal{H})$ is of class $\mathcal{Q}$, i.e., $T$ has the block matrix form (1) with $V, E$ and $Q$ satisfying (2)-(5). This question is discussed in the following example.

Example 7.3 Set $Y=\mathbb{T}_{+} \cup\left(\{1\} \times \mathbb{R}_{+}\right)$. Let $\Gamma$ be any nonempty compact subset of $Y$ such that

$$
\Gamma \cap(\{1\} \times(0, \infty)) \neq \emptyset .
$$

Set $\alpha=\max \left\{t \in \mathbb{R}_{+}:(1, t) \in \Gamma\right\}$. By (63), $\alpha>0$. It follows from Theorem 3.3 that there exists $T=\left[\begin{array}{ll}V & E \\ 0 & Q\end{array}\right] \in \mathcal{Q}_{\mathcal{H}_{1}, \mathcal{H}_{2}}$ such that

$$
\sigma(|Q|,|E|)=\Gamma \text {. }
$$

Since $\Gamma \subseteq Y$, we infer from Theorem 7.1 that $T$ is a quasi-Brownian isometry. According to (63) and Proposition 3.6, $T$ is not an isometry. Thus using [38, Proposition 5.1], we see that $T=\left[\begin{array}{ll}\tilde{V} & \tilde{E} \\ 0 & \tilde{Q}\end{array}\right] \in \mathcal{Q}_{\tilde{\mathcal{H}}_{1}, \tilde{\mathcal{H}}_{2}}$ relative to an orthogonal decomposition $\mathcal{H}=\tilde{\mathcal{H}}_{1} \oplus \tilde{\mathcal{H}}_{2}$, where $\tilde{Q}$ is an isometry. Consequently,

$$
\sigma(|\tilde{Q}|,|\tilde{E}|)=\sigma\left(I_{\tilde{\mathcal{H}}_{2}},|\tilde{E}|\right) \stackrel{(8)}{=}\{1\} \times \sigma(|\tilde{E}|) .
$$

In view of (63), (64) and Proposition 3.4 (see also Remark 3.5), we have

$$
\|T\|=r(|Q|,|E|)=r(|\tilde{Q}|,|\tilde{E}|)=\sqrt{1+\alpha^{2}},
$$

where

$$
\alpha=\max \left\{t \in \mathbb{R}_{+}:(1, t) \in \sigma(|Q|,|E|)\right\} .
$$

This, together with (65), implies that

$$
\alpha=\max \sigma(|\tilde{E}|)=\||\tilde{E}|\|=\|\tilde{E}\| .
$$

Since by (66), $(1, \alpha) \in \sigma(|Q|,|E|)$, we infer from (7) that $\alpha \in \sigma(|E|)$. Consequently,

$$
\|E\|=\||E|\|=\max \sigma(|E|) \geqslant \alpha \stackrel{(67)}{=}\|\tilde{E}\| .
$$

We now consider two important cases. First, if $\mathbb{T}_{+} \subseteq \Gamma$, then by (64) and (65) we obtain the two block matrix representations of $T$, namely 


$$
T=\left[\begin{array}{ll}
V & E \\
0 & Q
\end{array}\right] \in \mathcal{Q}_{\mathcal{H}_{1}, \mathcal{H}_{2}} \text { relative to } \mathcal{H}=\mathcal{H}_{1} \oplus \mathcal{H}_{2}
$$

and

$$
T=\left[\begin{array}{cc}
\tilde{V} & \tilde{E} \\
0 & \tilde{Q}
\end{array}\right] \in \mathcal{Q}_{\tilde{\mathcal{H}}_{1}, \tilde{\mathcal{H}}_{2}} \text { relative to } \mathcal{H}=\tilde{\mathcal{H}}_{1} \oplus \tilde{\mathcal{H}}_{2},
$$

such that

$$
Z \subseteq \sigma(|Q|,|E|) \quad \text { and } \quad Z \cap \sigma(|\tilde{Q}|,|\tilde{E}|)=\emptyset,
$$

where $Z:=\mathbb{T}_{+} \backslash\{(1,0)\}$. Second, if $\alpha>1$, then using (68), the inclusion $\Gamma \subseteq Y$ and (7), we deduce that $\alpha=\max \sigma(|E|)$; hence $\alpha=\||E|\|=\|E\|$ which by (67) yields $\alpha=\|E\|=\|\tilde{E}\|$.

\section{Brownian isometries of class $\mathcal{Q}$}

The aim of this section is to give a deeper insight into the structure of Brownian isometries of class $\mathcal{Q}$. We begin by proving two preparatory lemmata which are of some independent interest.

Lemma 8.1 Suppose $T=\left[\begin{array}{ll}V & E \\ 0 & Q\end{array}\right] \in \mathcal{Q}_{\mathcal{H}_{1}, \mathcal{H}_{2}}$. Then the operators $|Q|,|E|$ and $\left|Q^{*}\right|$ commute and the following conditions are equivalent:

(i) Tis a Brownian isometry,

(ii) $\left(|Q|^{2}-I\right)\left(|Q|^{2}+|E|^{2}-I\right)=0$ and $\left(\left|Q^{*}\right|^{2}-I\right)\left(|Q|^{2}+|E|^{2}-I\right)^{2}=0$.

Proof That the operators $|Q|,|E|$ and $\left|Q^{*}\right|$ commute can be deduced from (4) and (5) via the square root theorem (cf. Proposition 3.1(i)). Hence, by the equivalence (ii) $\Leftrightarrow$ (iii) of Theorem 7.1, it suffices to show that $\Delta_{T} \Delta_{T^{*}} \Delta_{T}=0$ if and only if $\left(\left|Q^{*}\right|^{2}-I\right)\left(|Q|^{2}+|E|^{2}-I\right)^{2}=0$. It is a routine matter to verify that

$$
\begin{aligned}
\Delta_{T} \Delta_{T^{*}} \Delta_{T} & =\left[\begin{array}{lc}
0 & 0 \\
0 & \left(\Omega_{1}-I\right)\left(\left|Q^{*}\right|^{2}-I\right)\left(\Omega_{1}-I\right)
\end{array}\right] \\
& =\left[\begin{array}{lc}
0 & 0 \\
0 & \left(\left|Q^{*}\right|^{2}-I\right)\left(\Omega_{1}-I\right)^{2}
\end{array}\right],
\end{aligned}
$$

where $\Omega_{1}=|Q|^{2}+|E|^{2}$. As a consequence, we get the desired equivalence.

Lemma 8.2 Suppose $T=\left[\begin{array}{ll}V & E \\ 0 & Q\end{array}\right] \in \mathcal{Q}_{\mathcal{H}_{1}, \mathcal{H}_{2}} \quad$ is a quasi-Brownian isometry. Let $\mathcal{H}_{2}=\mathcal{H}_{\mathrm{i}} \oplus \mathcal{H}_{\mathrm{si}}$ be an orthogonal decomposition of $\mathcal{H}_{2}$ (zero summands are allowed) satisfying the conditions $(a)$ and $(b)$ of Theorem 7.1 and let $\mathcal{H}_{\mathrm{i}}=\mathcal{H}_{\mathrm{u}} \oplus \mathcal{H}_{\mathrm{s}}$ be the von Neumann-Wold decomposition of $\mathcal{H}_{\mathrm{i}}$ for $\left.Q\right|_{\mathcal{H}_{\mathrm{i}}}$. Then $T$ is a Brownian isometry if and only if $\left.|E|\right|_{\mathcal{H}_{\mathrm{s}}}=0$. 
Proof Suppose $T$ is a Brownian isometry. Then by Lemma 8.1, we have

$$
0=\left.\left(I-\left|Q^{*}\right|^{2}\right)\left(|Q|^{2}+|E|^{2}-I\right)^{2}\right|_{\mathcal{H}_{\mathrm{s}}}=P A_{\mathrm{s}}^{4}=\left(P A_{\mathrm{s}}\right)^{4},
$$

where $A_{s}:=\left.|E|\right|_{\mathcal{H}_{\mathrm{s}}}$ and $P \in \boldsymbol{B}\left(\mathcal{H}_{\mathrm{s}}\right)$ is the orthogonal projection of $\mathcal{H}_{\mathrm{s}}$ onto $\mathscr{N}\left(Q_{\mathrm{s}}^{*}\right)$ with $Q_{\mathrm{s}}:=\left.Q\right|_{\mathcal{H}_{\mathrm{s}}}$ (by the moreover part of Theorem 7.1, $\mathcal{H}_{\mathrm{s}}$ reduces $Q$ and $|E|$ ). Because $\left(P A_{\mathrm{s}}\right)^{*}=P A_{\mathrm{s}}$, we infer from (69) that $P A_{\mathrm{s}}=0$. As a consequence, we see that $\mathscr{R}\left(A_{\mathrm{s}}\right) \subseteq \mathscr{N}\left(Q_{\mathrm{s}}^{*}\right)^{\perp}$. Since $Q_{\mathrm{s}}$ commutes with $A_{\mathrm{s}}$, so does $Q_{\mathrm{s}}^{*}$ and consequently $A_{\mathrm{s}}\left(\mathscr{N}\left(Q_{\mathrm{s}}^{*}\right)\right) \subseteq \mathscr{N}\left(Q_{\mathrm{s}}^{*}\right)$. Putting all of this together, we see that $A_{\mathrm{s}}\left(\mathscr{N}\left(Q_{\mathrm{s}}^{*}\right)\right)=\{0\}$. Therefore, because $Q$ commutes with $|E|$, we deduce that $|E| Q^{n} \mathscr{N}\left(Q_{\mathrm{s}}^{*}\right)=\{0\}$ for all $n \in \mathbb{Z}_{+}$. Since by (62), $\mathcal{H}_{\mathrm{s}}=\bigoplus_{n=0}^{\infty} Q^{n} \mathscr{N}\left(Q_{\mathrm{s}}^{*}\right)$, we conclude that $\left.|E|\right|_{\mathcal{H}_{\mathrm{s}}}=0$.

To prove the converse implication, assume that $|E|_{\mathcal{H}_{\mathrm{s}}}=0$. It follows from Theorem 7.1 that $\mathcal{H}_{2}=\mathcal{H}_{\mathrm{u}} \oplus \mathcal{H}_{\mathrm{s}} \oplus \mathcal{H}_{\mathrm{si}}$, the spaces $\mathcal{H}_{\mathrm{u}}, \mathcal{H}_{\mathrm{s}}$ and $\mathcal{H}_{\mathrm{si}}$ reduce both $Q$ and $|E|,\left.Q\right|_{\mathcal{H}_{\mathrm{u}}}$ is unitary, $\left.Q\right|_{\mathcal{H}_{\mathrm{s}}}$ is a unilateral shift and $\left(\left.Q\right|_{\mathcal{H}_{\text {si }}},|E|_{\mathcal{H}_{\mathrm{si}}}\right)$ is a spherical isometry. Now, straightforward calculations show that the condition (ii) of Lemma 8.1 holds. Hence by this lemma, $T$ is a Brownian isometry. This completes the proof.

We are now ready to characterize Brownian isometries of class $\mathcal{Q}$.

Theorem 8.3 Suppose $T=\left[\begin{array}{ll}V & E \\ 0 & Q\end{array}\right] \in \mathcal{Q}_{\mathcal{H}_{1}, \mathcal{H}_{2}}$. Then the following conditions are equivalent:

(i) $T$ is a Brownian isometry,

(ii) $\left(|Q|^{2}-I\right)\left(|Q|^{2}+|E|^{2}-I\right)=0$ and $\left(\left|Q^{*}\right|^{2}-I\right)\left(|Q|^{2}+|E|^{2}-I\right)=0$,

(iii) there exists an orthogonal decomposition $\mathcal{H}_{2}=\mathcal{H}_{\mathrm{u}} \oplus \mathcal{H}_{\mathrm{s}} \oplus \mathcal{H}_{\mathrm{si}}$ (zero summands are allowed) such that

(a) $\mathcal{H}_{\mathrm{u}}, \mathcal{H}_{\mathrm{s}}$ and $\mathcal{H}_{\mathrm{si}}$ reduce both $Q$ and $|E|$,

(b) $\left.Q\right|_{\mathcal{H}_{\mathrm{u}}}$ is a unitary operator and $\left.Q\right|_{\mathcal{H}_{\mathrm{s}}}$ is a unilateral shift (of finite or infinite multiplicity),

(c) $\left(\left.Q\right|_{\mathcal{H}_{\mathrm{si}}},|E|_{\mathcal{H}_{\mathrm{si}}}\right)$ is a spherical isometry,

(d) $\left.|E|\right|_{\mathcal{H}_{\mathrm{s}}}=0$.

Proof (i) $\Rightarrow$ (iii) Since any Brownian isometry is a quasi-Brownian isometry, it follows from Theorem 7.1 that there exists an orthogonal decomposition $\mathcal{H}_{2}=\mathcal{H}_{\mathrm{i}} \oplus \mathcal{H}_{\mathrm{si}}$ (zero summands are allowed) satisfying the conditions (a) and (b) of Theorem 7.1(v). Let $\mathcal{H}_{\mathrm{i}}=\mathcal{H}_{\mathrm{u}} \oplus \mathcal{H}_{\mathrm{s}}$ be the von Neumann-Wold decomposition of $\mathcal{H}_{\mathrm{i}}$ for $\left.Q\right|_{\mathcal{H}_{\mathrm{i}}}$. By the moreover part of Theorem 7.1, the orthogonal decomposition $\mathcal{H}_{2}=\mathcal{H}_{\mathrm{u}} \oplus \mathcal{H}_{\mathrm{s}} \oplus \mathcal{H}_{\mathrm{si}}$ satisfies the conditions (a), (b) and (c). Applying Lemma 8.2, we conclude that (d) holds.

(iii) $\Rightarrow$ (ii) This can be shown by straightforward calculations.

(ii) $\Rightarrow$ (i) This implication is a direct consequence of Lemma 8.1.

The following corollary is a consequence of Theorem 7.1, Lemma 8.2 and the uniqueness part of [49, Theorem I.1.1] (see also the proof of Theorem 8.3).

Corollary 8.4 Suppose $T=\left[\begin{array}{ll}V & E \\ 0 & Q\end{array}\right] \in \mathcal{Q}_{\mathcal{H}_{1}, \mathcal{H}_{2}}$. Then the following conditions are equivalent:

(i) T is a quasi-Brownian isometry which is not a Brownian isometry,

(ii) there exists an orthogonal decomposition $\mathcal{H}_{2}=\mathcal{H}_{\mathrm{u}} \oplus \mathcal{H}_{\mathrm{s}} \oplus \mathcal{H}_{\mathrm{si}}$ (zero summands are allowed) such that 
(a) $\mathcal{H}_{\mathrm{u}}, \mathcal{H}_{\mathrm{s}}$ and $\mathcal{H}_{\mathrm{si}}$ reduce both $Q$ and $|E|$,

(b) $\left.Q\right|_{\mathcal{H}_{\mathrm{u}}}$ is a unitary operator and $\left.Q\right|_{\mathcal{H}_{\mathrm{s}}}$ is a unilateral shift (of finite or infinite multiplicity),

(c) $\left(\left.Q\right|_{\mathcal{H}_{\mathrm{si}}},\left.|E|\right|_{\mathcal{H}_{\mathrm{si}}}\right)$ is a spherical isometry,

(d) $\left.|E|\right|_{\mathcal{H}_{\mathrm{s}}} \neq 0$.

As shown below, the class of Brownian isometries is the only subclass of $\mathcal{Q}$ considered in this paper which cannot be characterized by the Taylor spectrum $\sigma(|Q|,|E|)$ of the pair $(|Q|,|E|)$.

Remark 8.5 Notice that the condition (ii) of Theorem 8.3 is equivalent to the conjunction of the following two inclusions

$$
\begin{aligned}
\sigma(|Q|,|E|) & \subseteq \mathbb{T}_{+} \cup\left(\{1\} \times \mathbb{R}_{+}\right), \\
\sigma\left(|Q|,|E|,\left|Q^{*}\right|\right) & \subseteq\left(\mathbb{T}_{+} \times \mathbb{R}_{+}\right) \cup\left(\mathbb{R}_{+}^{2} \times\{1\}\right),
\end{aligned}
$$

where $\sigma\left(|Q|,|E|,\left|Q^{*}\right|\right)$ stands for the Taylor spectrum of $\left(|Q|,|E|,\left|Q^{*}\right|\right)$ (recall that the operators $|Q|,|E|$ and $\left|Q^{*}\right|$ commute; see Lemma 8.1). In view of Theorem 7.1, it remains to show that the equation $\left(\left|Q^{*}\right|^{2}-I\right)\left(|Q|^{2}+|E|^{2}-I\right)=0$ is equivalent to the second inclusion in (70). However, this is immediate from (6) and the spectral mapping theorem applied to the polynomial $p$ in three variables given by

$$
p(s, t, r)=\left(r^{2}-1\right)\left(s^{2}+t^{2}-1\right) .
$$

We conclude this remark by reexamining [7, Example 4.4]. Let $V \in \boldsymbol{B}\left(\mathcal{H}_{1}\right), E \in \boldsymbol{B}\left(\mathcal{H}_{2}, \mathcal{H}_{1}\right)$ and $Q \in \boldsymbol{B}\left(\mathcal{H}_{2}\right)$ be isometric operators such that $Q$ is not unitary and $V^{*} E=0$. As shown in [7, Example 4.4], the operator $T$ defined by (1) is a quasi-Brownian isometry (obviously of class $\mathcal{Q}$ ) which is not a Brownian isometry. Clearly, the first inclusion in (70) holds. Hence by the above discussion the second one does not hold. The latter also follows directly from the equality $\sigma\left(|Q|,|E|,\left|Q^{*}\right|\right)=\{1\} \times\{1\} \times\{0,1\}$ which is a consequence of the projection property of the Taylor spectrum. Regarding Corollary 8.4, note that $\mathcal{H}_{2}=\mathcal{H}_{\mathrm{u}} \oplus \mathcal{H}_{\mathrm{s}}$, $\mathcal{H}_{\mathrm{si}}=\{0\}, \mathcal{H}_{\mathrm{s}} \neq\{0\}$ and $\left.|E|\right|_{\mathcal{H}_{\mathrm{s}}} \neq 0$. Summarizing, the operator $T$ is a quasi-Brownian isometry which is not a Brownian isometry and $\sigma(|Q|,|E|)=\{(1,1)\}$. On the other hand, if $\tilde{T}$ is any nonisometric Brownian isometry, then it is a Brownian-type operator of class $\mathcal{U}$ (see the remark just after Definition 1.1), i.e., $\tilde{T}=\left[\begin{array}{cc}\tilde{V} & \tilde{E} \\ 0 & \tilde{Q}\end{array}\right] \in \mathcal{Q}_{\tilde{\mathcal{H}}_{1}, \tilde{\mathcal{H}}_{2}}$ relative to an orthogonal decomposition $\tilde{\mathcal{H}}_{1} \oplus \tilde{\mathcal{H}}_{2}$, where $\tilde{Q}$ is a unitary operator. As a consequence, $\sigma(|\tilde{Q}|,|\tilde{E}|)=\{(1,1)\}$. This means that Brownian isometries cannot be characterized by the Taylor spectrum $\sigma(|Q|,|E|)$.

\section{$9 m$-isometries and related operators of class $\mathcal{Q}$}

In this section we characterize $m$-contractions, $m$-isometries and $m$-expansions of class $\mathcal{Q}$ by using the Taylor spectrum approach.

Given an integer $m \geqslant 1$ and an operator $T \in \boldsymbol{B}(\mathcal{H})$, we write 


$$
\mathscr{B}_{m}(T)=\sum_{j=0}^{m}(-1)^{j}\left(\begin{array}{c}
m \\
j
\end{array}\right) T^{* j} T^{j} .
$$

Recall that an operator $T \in \boldsymbol{B}(\mathcal{H})$ is

- $m$-contractive if $\mathscr{B}_{m}(T) \geqslant 0$,

- m-expansive if $\mathscr{B}_{m}(T) \leqslant 0$,

- $m$-isometric if $T$ is $m$-contractive and $m$-expansive, that is $\mathscr{B}_{m}(T)=0$,

- completely hyperexpansive if $T$ is $m$-expansive for all $m \geqslant 1$.

The above-mentioned concepts can be attributed to many authors, such as Agler [1] (m-contractivity), Richter [42] (2-expansivity), Aleman [6] (complete hyperexpansivity for special operators), Agler [2] ( $m$-isometricity) and Athavale [11] ( $m$-expansivity and complete hyperexpansivity). It is well known that a 2-isometry is $m$-isometric for every integer $m \geqslant 2$ (see [3-5, Paper I, §1]). Combined with [42, Lemma 1(a)], this implies that each 2 -isometry is completely hyperexpansive. On the other hand, Agler proved in [1, Theorem 3.1] that an operator $T \in \boldsymbol{B}(\mathcal{H})$ is a subnormal contraction if and only if it is completely hypercontractive, i.e., $T$ is $m$-contractive for every positive integer $m$.

The expression $\mathscr{B}_{m}(T)$ for an operator $T$ of class $\mathcal{Q}$ can be described as follows.

Lemma 9.1 Suppose that $T=\left[\begin{array}{ll}V & E \\ 0 & Q\end{array}\right] \in \mathcal{Q}_{\mathcal{H}_{1}, \mathcal{H}_{2}}$. Then

$$
\mathscr{B}_{m}(T)=\left[\begin{array}{cc}
0 & 0 \\
0 & \psi_{m}(|Q|,|E|)
\end{array}\right], \quad m \in \mathbb{N},
$$

where $\psi_{m}: \mathbb{R}_{+}^{2} \rightarrow \mathbb{R}$ are polynomial functions defined by

$$
\psi_{m}(s, t)=\left(1-s^{2}-t^{2}\right)\left(1-s^{2}\right)^{m-1}, \quad(s, t) \in \mathbb{R}_{+}^{2}, m \in \mathbb{N} .
$$

Proof For $m \in \mathbb{N}$, we set $\Lambda_{m}=\sum_{j=0}^{m}(-1)^{j}\left(\begin{array}{c}m \\ j\end{array}\right) \Omega_{j}$, where $\Omega_{j}$ are as in (21). In view of Proposition 3.10(ii), we have

$$
\mathscr{B}_{m}(T)=\left[\begin{array}{cc}
0 & 0 \\
0 & \Lambda_{m}
\end{array}\right], \quad m \in \mathbb{N} .
$$

Let $G$ be the joint spectral measure of $(|Q|,|E|)$. It follows from (39) and (40) that

$$
\Lambda_{m}=\int_{\mathbb{R}_{+}^{2}} \tilde{\psi}_{m} d G, \quad m \in \mathbb{N},
$$

where $\tilde{\psi}_{m}: \mathbb{R}_{+}^{2} \rightarrow \mathbb{R}$ are continuous functions defined by

$$
\tilde{\psi}_{m}(s, t)=\sum_{j=0}^{m}(-1)^{j}\left(\begin{array}{c}
m \\
j
\end{array}\right) \varphi_{j}(s, t), \quad(s, t) \in \mathbb{R}_{+}^{2}, m \in \mathbb{N} .
$$

Now we show that $\tilde{\psi}_{m}=\psi_{m}$ for any $m \in \mathbb{N}$. For this, note that 


$$
\tilde{\psi}_{m}(1, t) \stackrel{(40)}{=} t^{2} \sum_{j=1}^{m}(-1)^{j}\left(\begin{array}{c}
m \\
j
\end{array}\right) j=-m t^{2} \sum_{j=0}^{m-1}(-1)^{j}\left(\begin{array}{c}
m-1 \\
j
\end{array}\right), \quad t \in \mathbb{R}_{+}, m \in \mathbb{N}
$$

Hence, we get

$$
\tilde{\psi}_{m}(1, t)=\left\{\begin{array}{ll}
-t^{2} & \text { if } m=1, \\
0 & \text { if } m \geqslant 2,
\end{array} \quad t \in \mathbb{R}_{+} .\right.
$$

In turn if $s \neq 1$, we can argue as follows:

$$
\begin{aligned}
\tilde{\psi}_{m}(s, t) & \stackrel{(41)}{=} \sum_{j=0}^{m}(-1)^{j}\left(\begin{array}{c}
m \\
j
\end{array}\right)\left(\frac{t^{2}}{1-s^{2}}+\left(1-\frac{t^{2}}{1-s^{2}}\right) s^{2 j}\right) \\
& =\left(1-\frac{t^{2}}{1-s^{2}}\right) \sum_{j=0}^{m}(-1)^{j}\left(\begin{array}{c}
m \\
j
\end{array}\right) s^{2 j} \\
& =\left(1-s^{2}-t^{2}\right)\left(1-s^{2}\right)^{m-1}, \quad(s, t) \in\left(\mathbb{R}_{+} \backslash\{1\}\right) \times \mathbb{R}_{+}, m \in \mathbb{N} .
\end{aligned}
$$

Putting all this together we see that $\tilde{\psi}_{m}=\psi_{m}$ for all $m \in \mathbb{N}$. Combined with (9), (72) and (73), this completes the proof.

We are now in a position to characterize $m$-contractivity, $m$-isometricity and $m$-expansivity of operators of class $\mathcal{Q}$. The spectral regions for $m$-contractivity and $m$-expansivity of operators of class $\mathcal{Q}$ are illustrated in Figs. 2 and 3 (for the case $m=1$, see Proposition 3.6).

Theorem 9.2 Assume that $T=\left[\begin{array}{ll}V & E \\ 0 & Q\end{array}\right] \in \mathcal{Q}_{\mathcal{H}_{1}, \mathcal{H}_{2}}$ and $m \geqslant 2$ is an integer. Then the following assertions hold:

(i) $T$ is m-contractive if and only if

$$
\sigma(|Q|,|E|) \subseteq \begin{cases}\overline{\mathbb{D}}_{+} \cup\left(\{1\} \times \mathbb{R}_{+}\right) & \text {if } m \text { is odd } \\ \overline{\mathbb{D}}_{+} \cup\left([1, \infty) \times \mathbb{R}_{+}\right) & \text {if } m \text { is even }\end{cases}
$$

(ii) $T$ is m-expansive if and only if

$$
\sigma(|Q|,|E|) \subseteq \begin{cases}\mathbb{R}_{+}^{2} \backslash \mathbb{D}_{+} \\ \left(\mathbb{R}_{+}^{2} \backslash \mathbb{D}_{+}\right) \cap\left([0,1] \times \mathbb{R}_{+}\right) \text {if } m \text { is is even, }\end{cases}
$$

(iii) $T$ is m-isometric if and only if $\sigma(|Q|,|E|) \subseteq \mathbb{T}_{+} \cup\left(\{1\} \times \mathbb{R}_{+}\right)$.

Proof Since the proofs of (i) and (ii) are similar, we justify only (i). Let $G$ be the joint spectral measure of the pair $(|Q|,|E|)$. Observe that by (9) and Lemma $9.1, \mathscr{B}_{m}(T) \geqslant 0$ if and only if $\int_{\mathbb{R}_{+}^{2}} \psi_{m} d G \geqslant 0$. By Theorem 2.1(i) and Lemma 4.9, the latter holds if and only if

$$
\sigma(|Q|,|E|)=\operatorname{supp} G \subseteq\left\{(s, t) \in \mathbb{R}_{+}^{2}: \psi_{m}(s, t) \geqslant 0\right\} .
$$

Using (71), we verify that 

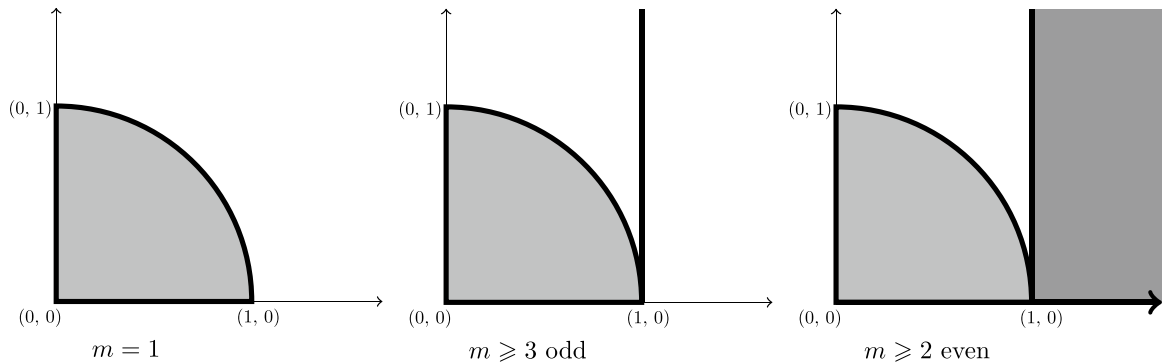

Fig. 2 Spectral region for $m$-contractivity of operators of class $\mathcal{Q}$
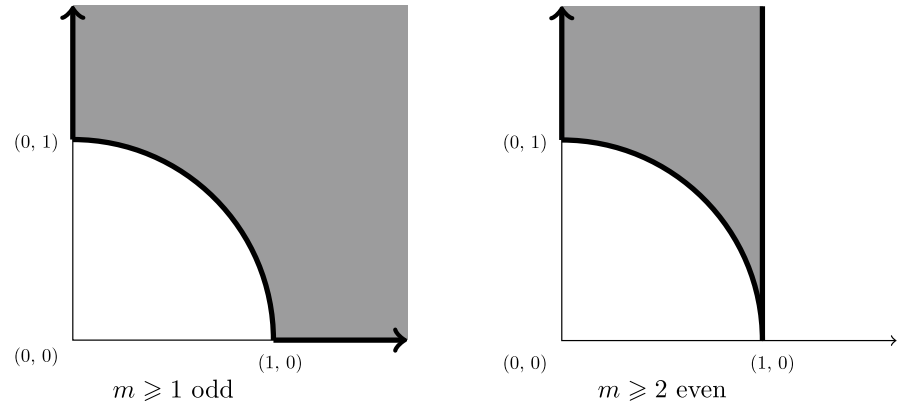

Fig. 3 Spectral region for $m$-expansivity of operators of class $\mathcal{Q}$

$$
\left\{(s, t) \in \mathbb{R}_{+}^{2}: \psi_{m}(s, t) \geqslant 0\right\}= \begin{cases}\overline{\mathbb{D}}_{+} \cup\left(\{1\} \times \mathbb{R}_{+}\right) & \text {if } m \text { is odd } \\ \overline{\mathbb{D}}_{+} \cup\left([1, \infty) \times \mathbb{R}_{+}\right) & \text {if } m \text { is even }\end{cases}
$$

Combined with (74), this yields (i). Finally, (iii) can be deduced from (i) and (ii). This completes the proof.

Corollary 9.3 Assume that $T=\left[\begin{array}{ll}V & E \\ 0 & Q\end{array}\right] \in \mathcal{Q}_{\mathcal{H}_{1}, \mathcal{H}_{2}}$ and $m \geqslant 2$ is an integer. Then the following assertions hold:

(i) if $m$ is odd (resp., even), then $T$ is $m$-contractive if and only if $T$ is 3-contractive (resp., 2-contractive),

(ii) if $m$ is odd (resp., even), then $T$ is m-expansive if and only if $T$ is expansive (resp., 2-expansive),

(iii) $T$ is $m$-isometric if and only if $T$ is 2-isometric,

(iv) $T$ is completely hypercontractive if and only if $T$ is contractive,

(v) $T$ is completely hyperexpansive if and only if $T$ is 2-expansive.

Proof Use Theorem 9.2 and additionally Proposition 3.6 in the cases (ii), (iv) and (v).

The example below illustrates Theorem 9.2. 
Example 9.4 (Example 6.4 continued) Let $T_{\tau, \eta}$ be as in Example 6.4. Assume that $m \geqslant 2$. Using (55), Proposition 3.6 and Theorem 9.2, we get the following:

$1^{\circ} \quad$ if $m$ is odd, then the operator $T_{\tau, \eta}$ is $m$-contractive if and only if $\tilde{Q}$ is contractive and $(|\tau|,|\eta|) \in \overline{\mathbb{D}}_{+} \cup\left(\{1\} \times \mathbb{R}_{+}\right)$,

$2^{\circ}$ if $m$ is even, then the operator $T_{\tau, \eta}$ is $m$-contractive if and only if $(|\tau|,|\eta|) \in \overline{\mathbb{D}}_{+} \cup\left([1, \infty) \times \mathbb{R}_{+}\right)$,

$3^{\circ}$ if $m$ is odd, then the operator $T_{\tau, \eta}$ is $m$-expansive if and only if $T_{\tau, \eta}$ is expansive, or equivalently if and only if $\tilde{Q}$ is expansive and $(|\tau|,|\eta|) \in \mathbb{R}_{+}^{2} \backslash \mathbb{D}_{+}$,

$4^{\circ}$ if $m$ is even, then the operator $T_{\tau, \eta}$ is $m$-expansive if and only if $\tilde{Q}$ is an isometry and $(|\tau|,|\eta|) \in\left(\mathbb{R}_{+}^{2} \backslash \mathbb{D}_{+}\right) \cap\left([0,1] \times \mathbb{R}_{+}\right)$,

$5^{\circ}$ the operator $T_{\tau, \eta}$ is $m$-isometric if and only if $\tilde{Q}$ is an isometry and $(|\tau|,|\eta|) \in \mathbb{T}_{+} \cup\left(\{1\} \times \mathbb{R}_{+}\right)$,

$6^{\circ}$ the operator $T_{\tau, \eta}$ is completely hypercontractive if and only if $\tilde{Q}$ is a contraction and $(|\tau|,|\eta|) \in \overline{\mathbb{D}}_{+}$,

$7^{\circ}$ the operator $T_{\tau, \eta}$ is completely hyperexpansive if and only if $\tilde{Q}$ is an isometry and $(|\tau|,|\eta|) \in\left(\mathbb{R}_{+}^{2} \backslash \mathbb{D}_{+}\right) \cap\left([0,1] \times \mathbb{R}_{+}\right)$.

Remark 9.5 Note that in view of [29, Theorem 2.5], if $m \geqslant 2$ is even, then any $m$-expansive operator is $(m-1)$-expansive, while if $m \geqslant 3$ is odd, then any $m$-contractive operator is $(m-1)$-contractive. Using the assertions $1^{\circ}-4^{\circ}$ of Example 9.4 one can easily show that none of these implications can be reversed. It is well known that quasi-Brownian isometries are 2-isometric, 2-isometries are completely hyperexpansive, complete hyperexpansions are 2-expansive, and finally 2-expansions are expansive (see [42, Lemma 1]). In general, none of these implications can be reversed. Using Remark 8.5, Theorem 7.1, Corollary 9.3 and Example 9.4, one can show that in the class $\mathcal{Q}$, these relations take the following form:

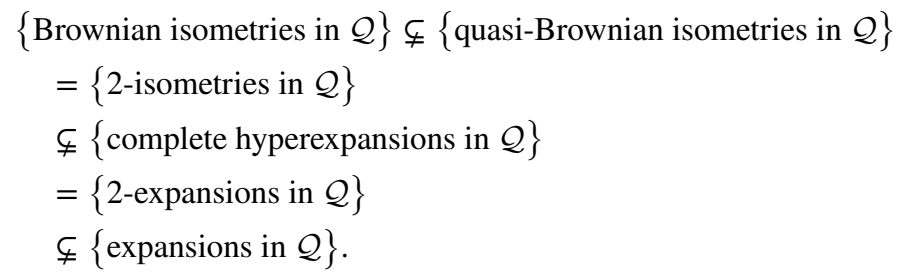

We refer the reader to Fig. 4 describing spectral regions for the above-mentioned subclasses of the class $\mathcal{Q}$ (except for Brownian isometries, cf. Remark 8.5).

A recent result due to Badea and Suciu (see [12, Theorem 3.4]), which states that a $\Delta_{T}$-regular 2-expansive operator $T$ is completely hyperexpansive if and only if its Cauchy dual $T^{\prime}$ is subnormal, solves in the affirmative the Cauchy dual subnormality problem in the class of $\Delta_{T}$-regular 2-expansions (see [7, Theorem 4.5] for an earlier solution of this problem in the class of $\Delta_{T}$-regular 2-isometries). It is well known and easy to prove that the relation $T \longleftrightarrow T^{\prime}$ is a one-to-one correspondence between expansive operators and left-invertible contractions. When restricted to operators of class $\mathcal{Q}$, this correspondence becomes a bijection between expansions and left-invertible subnormal contractions (see Corollary 6.2). In view of Proposition 3.10(v), expansions $T$ of class $\mathcal{Q}$ are always $\Delta_{T}$ -regular. This suggests that there may exist $\Delta_{T}$-regular operators outside of the class of completely hyperexpansive ones for which the Cauchy dual subnormality problem has an 


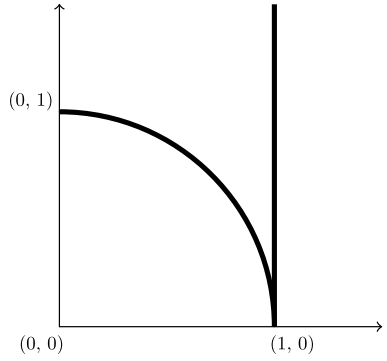

quasi-Brownian isometries 2-isometries

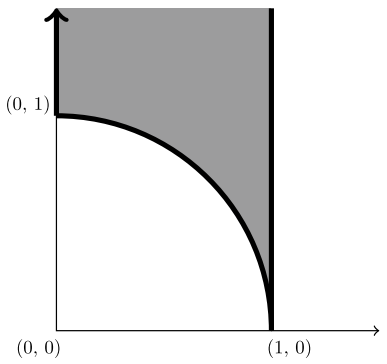

complete hyperexpansions 2-expansions

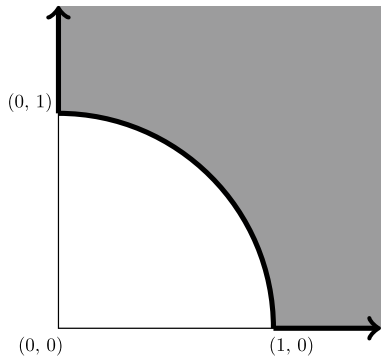

expansions

Fig. 4 Spectral regions for some subclasses of the class $\mathcal{Q}$

affirmative solution. This is really the case as shown in Example 9.7 below which is based on Proposition 9.6. The proposition itself is a direct consequence of Propositions 3.6 and 3.10(v), Corollary 6.2 and Theorem 9.2(ii).

Proposition 9.6 If $T=\left[\begin{array}{cc}V & E \\ 0 & Q\end{array}\right] \in \mathcal{Q}_{\mathcal{H}_{1}, \mathcal{H}_{2}}$ is such that $\sigma(|Q|,|E|) \subseteq \mathbb{R}_{+}^{2} \backslash \mathbb{D}_{+}$and $\sigma(|Q|,|E|) \cap\left((1, \infty) \times \mathbb{R}_{+}\right) \neq \emptyset$, then $T$ is a $\Delta_{T}$-regular expansion which is not 2-expansive (so not completely hyperexpansive) and whose Cauchy dual $T^{\prime}$ is a subnormal contraction.

To have a concrete example of an operator satisfying the assumptions of Proposition 9.6, we revisit Example 6.4 again.

Example 9.7 (Example 6.4 continued) Let $T_{\tau, \eta}$ be as in Example 6.4. Suppose that $\tilde{Q}$ is expansive and $(|\tau|,|\eta|) \in(1, \infty) \times(0, \infty)$. Applying (6) and (55), we see that the operator $T_{\tau, \eta}$ satisfies the hypothesis of Proposition 9.6. As a consequence, $T_{\tau, \eta}$ is a $\Delta_{T_{\tau, \eta}}$-regular expansion of class $\mathcal{Q}$ such that the Cauchy dual $T_{\tau, \eta}^{\prime}$ of $T_{\tau, \eta}$ is a subnormal contraction but $T_{\tau, \eta}$ itself is not 2-expansive (so not completely hyperexpansive).

\section{Linear operator pencils built over the class $\mathcal{Q}$}

In this section we study linear operator pencils that are associated with operators of class $\mathcal{Q}$. By a linear operator pencil (see [28]) we mean a mapping

$$
\Psi: \mathbb{C} \ni \lambda \longmapsto A+\lambda B \in \boldsymbol{B}(\mathcal{H}),
$$

where $A, B \in \boldsymbol{B}(\mathcal{H})$. Given $T=\left[\begin{array}{cc}V & E \\ 0 & Q\end{array}\right] \in \mathcal{Q}_{\mathcal{H}_{1}, \mathcal{H}_{2}}$, we define the linear operator pencil $T^{\dagger}(\lambda)$ by

$$
T^{\dagger}(\lambda)=\left[\begin{array}{cc}
V & \lambda E \\
0 & Q
\end{array}\right]=\left[\begin{array}{ll}
V & 0 \\
0 & Q
\end{array}\right]+\lambda\left[\begin{array}{ll}
0 & E \\
0 & 0
\end{array}\right], \quad \lambda \in \mathbb{C} .
$$

Clearly, $T^{\dagger}(\lambda) \in \mathcal{Q}_{\mathcal{H}_{1}, \mathcal{H}_{2}}$ for every $\lambda \in \mathbb{C}$. Observe that $T^{\dagger}(\lambda)$ can be regarded as the perturbation of the quasinormal operator $\left[\begin{array}{ll}V & 0 \\ 0 & Q\end{array}\right]$ by the nilpotent operator $\lambda\left[\begin{array}{ll}0 & E \\ 0 & 0\end{array}\right]$. It is worth pointing out that the operators $\left[\begin{array}{ll}V & 0 \\ 0 & Q\end{array}\right]$ and $\left[\begin{array}{ll}0 & E \\ 0 & 0\end{array}\right]$ do not commute in general (they commute if and 
only if $V E=E Q$.) Note that by Corollary $5.1, T^{\dagger}(\lambda)$ is subnormal if and only if $T^{\dagger}(|\lambda|)$ is subnormal. This justifies why we concentrate on describing the set $\mathscr{S}^{\dagger}(T)$ given by

$$
\mathscr{S}^{\dagger}(T)=\left\{\alpha \in \mathbb{R}_{+}: T^{\dagger}(\alpha) \text { is subnormal }\right\} .
$$

If, moreover, $E \neq 0$ and $\sigma_{\sharp}(|Q|,|E|) \subseteq[0,1] \times(0, \infty)$, then we define $\beta^{\dagger}(T) \in \mathbb{R}_{+}$by (cf. (38))

$$
\beta^{\dagger}(T)=\inf _{(s, t) \in \sigma_{\sharp}(|Q|,|E|)} \sqrt{\frac{1-s^{2}}{t^{2}}} .
$$

Using $\beta^{\dagger}(T)$ we can describe the set $\mathscr{S}^{\dagger}(T)$ explicitly.

Theorem 10.1 Suppose that $T=\left[\begin{array}{ll}V & E \\ 0 & Q\end{array}\right] \in \mathcal{Q}_{\mathcal{H}_{1}, \mathcal{H}_{2}}$ and $E \neq 0$. Then the following assertions hold:

(i) $0 \in \mathscr{S}^{\dagger}(T)$,

(ii) $\mathscr{S}^{\dagger}(T) \backslash\{0\} \neq \emptyset$ if and only if $\sigma_{\sharp}(|Q|,|E|) \subseteq[0,1] \times(0, \infty)$ and $\beta^{\dagger}(T)>0$,

(iii) if $\mathscr{S}^{\dagger}(T) \backslash\{0\} \neq \emptyset$, then

$$
\mathscr{S}^{\dagger}(T)=\left[0, \beta^{\dagger}(T)\right] \text {. }
$$

Moreover, if $\sigma_{\sharp}(|Q|,|E|) \subseteq[0,1] \times(0, \infty)$, then

$$
\mathscr{S}^{\dagger}(T)=\{0\} \Longleftrightarrow \beta^{\dagger}(T)=0 .
$$

Proof First observe that $T^{\dagger}(0)$ is a quasinormal operator and thus by [19, Proposition II.1.7], $T^{\dagger}(0)$ is subnormal, which yields (i). In view of (i) and Theorems 2.1(iii) and 1.2(ii), we have

$$
\mathscr{S}^{\dagger}(T)=\{0\} \cup\left\{\alpha \in(0, \infty): s^{2}+\alpha^{2} t^{2} \leqslant 1, \forall(s, t) \in \sigma_{\sharp}(|Q|,|E|)\right\} .
$$

It is now a routine matter to show that for any $\alpha \in \mathscr{S}^{\dagger}(T),[0, \alpha] \subseteq \mathscr{S}^{\dagger}(T)$.

(ii) and (iii) Suppose that $\alpha \in \mathscr{S}^{\dagger}(T) \backslash\{0\}$. Then by (77), we have

$$
\alpha^{2} \leqslant \frac{1-s^{2}}{t^{2}}, \quad(s, t) \in \sigma_{\sharp}(|Q|,|E|) .
$$

As a consequence, $\sigma_{\sharp}(|Q|,|E|) \subseteq[0,1] \times(0, \infty)$ and $0<\sup \mathscr{S}^{\dagger}(T) \leqslant \beta^{\dagger}(T)$. Clearly, by (77), $\beta^{\dagger}(T) \in \mathscr{S}^{\dagger}(T) \backslash\{0\}$. Hence, in view of the discussion in the previous paragraph, (76) holds.

In turn, if $\sigma_{\sharp}(|Q|,|E|) \subseteq[0,1] \times(0, \infty)$ and $\beta^{\dagger}(T)>0$, then as above we verify that $\beta^{\dagger}(T) \in \mathscr{S}^{\dagger}(T) \backslash\{0\}$.

The "moreover" part follows from (i) and (ii). This completes the proof.

There is another possibility of associating a linear operator pencil with an operator of class $\mathcal{Q}$. Namely, given $T=\left[\begin{array}{cc}V & E \\ 0 & Q\end{array}\right] \in \mathcal{Q}_{\mathcal{H}_{1}, \mathcal{H}_{2}}$, we define the pencil $T_{\dagger}(\cdot)$ by 


$$
T_{\dagger}(\lambda)=\left[\begin{array}{cc}
V & E \\
0 & \lambda Q
\end{array}\right]=\left[\begin{array}{ll}
V & E \\
0 & 0
\end{array}\right]+\lambda\left[\begin{array}{ll}
0 & 0 \\
0 & Q
\end{array}\right], \quad \lambda \in \mathbb{C},
$$

and the corresponding set $\mathscr{S}_{\dagger}(T)$ by

$$
\mathscr{S}_{\dagger}(T)=\left\{\alpha \in \mathbb{R}_{+}: T_{\dagger}(\alpha) \text { is subnormal }\right\} .
$$

As before, by Corollary 5.1, $T_{\dagger}(\lambda)$ is subnormal if and only if $T_{\dagger}(|\lambda|)$ is subnormal, so we can concentrate on describing the set $\mathscr{S}_{\dagger}(T)$. Obviously, $T_{\dagger}(\lambda) \in \mathcal{Q}_{\mathcal{H}_{1}, \mathcal{H}_{2}}$ for every $\lambda \in \mathbb{C}$. The operator $T_{\dagger}(\lambda)$ can be regarded as the perturbation of $\left[\begin{array}{ll}V & E \\ 0 & 0\end{array}\right]$ by the quasinormal operator $\lambda\left[\begin{array}{ll}0 & 0 \\ 0 & Q\end{array}\right]$ (in view of Theorem 10.2(i), $\left[\begin{array}{ll}V & E \\ 0 & 0\end{array}\right]$ is subnormal provided $\mathscr{S}_{\dagger}(T) \neq \emptyset$ ). Note also that

$$
\left[\begin{array}{ll}
V & E \\
0 & 0
\end{array}\right] \text { and }\left[\begin{array}{ll}
0 & 0 \\
0 & Q
\end{array}\right] \text { commute } \Longleftrightarrow E Q=0 \Longleftrightarrow|Q \| E|=0 \text {. }
$$

Indeed, the former equivalence is a consequence of straightforward calculations, while the latter follows from the identities:

$$
(|Q \| E|)^{2} \stackrel{(*)}{=} Q^{*} Q E^{*} E \stackrel{(4)}{=}(E Q)^{*} E Q,
$$

where $(*)$ is a consequence of Proposition 3.1(i).

We are now in a position to describe the set $\mathscr{S}_{\dagger}(T)$.

Theorem 10.2 Suppose that $T=\left[\begin{array}{ll}V & E \\ 0 & Q\end{array}\right] \in \mathcal{Q}_{\mathcal{H}_{1}, \mathcal{H}_{2}} \cdot$ Set

$$
\sigma_{b}(|Q|,|E|)=\sigma(|Q|,|E|) \cap((0, \infty) \times(0, \infty)) .
$$

Then the following assertions hold:

(i) $\mathscr{S}_{\dagger}(T) \neq \emptyset$ if and only if $\|E\| \leqslant 1$,

(ii) if $\|E\| \leqslant 1$ and $\sigma_{b}(|Q|,|E|)=\emptyset$, then $\mathscr{S}_{\dagger}(T)=\mathbb{R}_{+}$,

(iii) if $\|E\| \leqslant 1$ and $\sigma_{b}(|Q|,|E|) \neq \emptyset$, then $\mathscr{S}_{\dagger}(T)=\left[0, \beta_{\dagger}(T)\right]$, where ${ }^{5}$

$$
\beta_{\dagger}(T):=\inf _{(s, t) \in \sigma_{b}(|Q|,|E|)} \sqrt{\frac{1-t^{2}}{s^{2}}} .
$$

Proof It follows from Theorems 2.1(iii) and 1.2(ii) that

$$
\mathscr{S}_{\dagger}(T)=\left\{\alpha \in \mathbb{R}_{+}: \alpha^{2} s^{2}+t^{2} \leqslant 1, \forall(s, t) \in \sigma_{\sharp}(|Q|,|E|)\right\} .
$$

Recall that the set $\sigma_{\sharp}(|Q|,|E|)$ may be empty (see (38)). It is easily seen that

$$
[0, \alpha] \subseteq \mathscr{S}_{\dagger}(T) \text { whenever } \alpha \in \mathscr{S}_{\dagger}(T) .
$$

(i) In view of (38) and (80), there is no loss of generality in assuming that $\sigma_{\sharp}(|Q|,|E|) \neq \emptyset$. Suppose that $\mathscr{S}_{\dagger}(T) \neq \emptyset$. Then, by (7) and $(80), \sigma(|E|) \backslash\{0\} \subseteq[0,1]$, hence by (6), $\|E\| \leqslant 1$. Conversely, if $\|E\| \leqslant 1$, then by (6), (7) and (80), $0 \in \mathscr{S}_{\dagger}(T)$.

\footnotetext{
${ }^{5}$ It follows from $\|E\| \leqslant 1,(6)$ and $(7)$ that $\sigma_{b}(|Q|,|E|) \subseteq(0, \infty) \times[0,1]$, which implies that $\beta_{\dot{\dagger}}(T)$ is well defined.
} 
(ii) Since $\|E\| \leqslant 1$, we infer from (6) that $\sigma(|E|) \subseteq[0,1]$. Hence, if $(s, t) \in \sigma_{\sharp}(|Q|,|E|) \cap\left(\{0\} \times \mathbb{R}_{+}\right)$, then by using (7) we see that $\alpha^{2} s^{2}+t^{2} \leqslant 1$ for all $\alpha \in \mathbb{R}_{+}$. By assumption

$$
\sigma_{\sharp}(|Q|,|E|) \cap\left((0, \infty) \times \mathbb{R}_{+}\right)=\sigma_{b}(|Q|,|E|)=\emptyset,
$$

and consequently, by $(80), \mathscr{S}_{\dagger}(T)=\mathbb{R}_{+}$.

(iii) Suppose that $\|E\| \leqslant 1$ and $\sigma_{b}(|Q|,|E|) \neq \emptyset$. If $\alpha \in \mathscr{S}_{\dagger}(T)$, then by (80), we have

$$
\alpha^{2} \leqslant \frac{1-t^{2}}{s^{2}}, \quad(s, t) \in \sigma_{b}(|Q|,|E|),
$$

which implies that sup $\mathscr{S}_{\dagger}(T) \leqslant \beta_{\dagger}(T)$. Now we prove the opposite inequality. As in (ii), we see that if $(s, t) \in \sigma_{\sharp}(|Q|,|E|) \cap\left(\{0\} \times \mathbb{R}_{+}\right)$, then $\alpha^{2} s^{2}+t^{2} \leqslant 1$ for all $\alpha \in \mathbb{R}_{+}$. In turn, if

$$
(s, t) \in \sigma_{\sharp}(|Q|,|E|) \cap\left((0, \infty) \times \mathbb{R}_{+}\right)=\sigma_{b}(|Q|,|E|),
$$

then the inequality $\alpha^{2} s^{2}+t^{2} \leqslant 1$ holds for $\alpha=\beta_{\dagger}(T)$. Therefore, by $(80), \beta_{\dagger}(T) \in \mathscr{S}_{\dagger}(T)$. Combined with (81), this implies that $\mathscr{S}_{\dagger}(T)=\left[0, \beta_{\dagger}(T)\right]$, which completes the proof.

Remark 10.3 Concerning Theorem 10.2, it is worth mentioning that according to the assertions (11) and (78) we have

$$
\sigma_{b}(|Q|,|E|)=\emptyset \Longleftrightarrow|Q||E|=0 \Longleftrightarrow\left[\begin{array}{ll}
V & E \\
0 & 0
\end{array}\right] \text { and }\left[\begin{array}{ll}
0 & 0 \\
0 & Q
\end{array}\right] \text { commute. }
$$

In other words, the set $\sigma_{b}(|Q|,|E|)$ is empty if and only if the perturbation $T_{\dagger}(\lambda)$ of $\left[\begin{array}{ll}V & E \\ 0 & 0\end{array}\right]$ commutes with the perturbing operator $\lambda\left[\begin{array}{ll}0 & 0 \\ 0 & Q\end{array}\right]$ for some $\lambda \in \mathbb{C} \backslash\{0\}$.

We now show that for an arbitrary $b \in \mathbb{R}_{+}$, there exists $T=\left[\begin{array}{ll}V & E \\ 0 & Q\end{array}\right] \in \mathcal{Q}_{\mathcal{H}_{1}, \mathcal{H}_{2}}$ such that $\mathscr{S}^{\dagger}(T)=[0, b]$. Similarly, for a given $b \in \mathbb{R}_{+} \cup\{\infty\}$, we can find $T=\left[\begin{array}{cc}V & E \\ 0 & Q\end{array}\right] \in \mathcal{Q}_{\mathcal{H}_{1}, \mathcal{H}_{2}}$ such that $\mathscr{S}_{\dagger}(T)=[0, b] \cap \mathbb{R}_{+}$.

Example 10.4 (Example 6.4 continued) Let $T_{\tau, \eta}$ be as in Example 6.4. We begin by showing that for every $b \in \mathbb{R}_{+}$, there exist $\tau \in \mathbb{C}$ and $\eta \in \mathbb{C} \backslash\{0\}$ such that $\mathscr{S}^{\dagger}\left(T_{\tau, \eta}\right)=[0, b]$. Indeed, it follows from (55) that $\sigma_{\sharp}\left(\left|Q_{\tau}\right|,\left|E_{\eta}\right|\right)=\{(|\tau|,|\eta|)\}$. Assume additionally that $|\tau| \leqslant 1$. Combined with (75), this gives

$$
\beta^{\dagger}\left(T_{\tau, \eta}\right)=\sqrt{\frac{1-|\tau|^{2}}{|\eta|^{2}}} .
$$

First, suppose that $b=0$. Then by considering the case $|\tau|=1$ we infer from the moreover part of Theorem 10.1 that $\mathscr{S}^{\dagger}\left(T_{\tau, \eta}\right)=[0, b]$. Let now $b>0$. Then by taking into account the case $|\tau|<1$ we deduce from (82) and Theorem 10.1 that $\mathscr{S}^{\dagger}\left(T_{\tau, \eta}\right)=\left[0, \beta^{\dagger}\left(T_{\tau, \eta}\right)\right]$. This together with (82) shows that there exists $\eta \in \mathbb{C} \backslash\{0\}$ such that $\beta^{\dagger}\left(T_{\tau, \eta}\right)=b$.

Similarly, using Theorem 10.2, one can show that for every $b \in \mathbb{R}_{+} \cup\{\infty\}$, there exist parameters $\tau$ and $\eta$ such that $\mathscr{S}_{\dagger}\left(T_{\tau, \eta}\right)=[0, b] \cap \mathbb{R}_{+}$. We leave the details to the reader.

We conclude this paper by commenting the contents of this section. In view of Theorem 9.2, the technique of using the Taylor spectrum developed here can also be applied to describe the sets of the form 


$$
\left\{\alpha \in \mathbb{R}_{+}: T^{\dagger}(\alpha) \text { is in } \mathscr{C}\right\} \text { and }\left\{\alpha \in \mathbb{R}_{+}: T_{\dagger}(\alpha) \text { is in } \mathscr{C}\right\} \text {, }
$$

where $\mathscr{C}$ is one of the classes of operators appearing in Sect. 9 including $m$-contractions, $m$-expansions, etc. As the number of cases to be considered is large (in particular depends on the parity of $m$ ) and each of them requires separate treatment, we decided not to include details in this paper.

Acknowledgements A part of this paper was written while the first and third authors visited Jagiellonian University in Summer of 2019, and the second and fourth authors visited Kyungpook National University in Autumn of 2019. They wish to thank the faculties and the administrations of these units for their warm hospitality.

Funding The research of the third author was supported by the National Research Foundation of Korea (NRF) grant funded by the Korea Government (MSIT) (2018R1A2B6003660).

Open Access This article is licensed under a Creative Commons Attribution 4.0 International License, which permits use, sharing, adaptation, distribution and reproduction in any medium or format, as long as you give appropriate credit to the original author(s) and the source, provide a link to the Creative Commons licence, and indicate if changes were made. The images or other third party material in this article are included in the article's Creative Commons licence, unless indicated otherwise in a credit line to the material. If material is not included in the article's Creative Commons licence and your intended use is not permitted by statutory regulation or exceeds the permitted use, you will need to obtain permission directly from the copyright holder. To view a copy of this licence, visit http://creativecommons.org/licenses/by/4.0/.

\section{References}

1. Agler, J.: Hypercontractions and subnormality. J. Oper. Theory 13, 203-217 (1985)

2. Agler, J.: A disconjugacy theorem for Toeplitz operators. Am. J. Math. 112, 1-14 (1990)

3. Agler, J., Stankus, M.: $m$-isometric transformations of Hilbert spaces, I. Integr. Equ. Oper. Theory 21, 383-429 (1995)

4. Agler, J., Stankus, M.: $m$-isometric transformations of Hilbert spaces, II. Integr. Equ. Oper. Theory 23, $1-48(1995)$

5. Agler, J., Stankus, M.: $m$-isometric transformations of Hilbert spaces, III. Integr. Equ. Oper. Theory 24, 379-421 (1996)

6. Aleman, A.: The multiplication operator on Hilbert spaces of analtyic functions. Habilitationsschrift, Fern-Universität Hagen (1993)

7. Anand, A., Chavan, S., Jabłoński, Z.J., Stochel, J.: A solution to the Cauchy dual subnormality problem for 2-isometries. J. Funct. Anal. 277, 108292 (2019)

8. Anand, A., Chavan, S., Jabłoński, Z.J., Stochel, J.: Complete systems of unitary invariants for some classes of 2-isometries. Banach J. Math. Anal. 13, 359-385 (2019)

9. Ash, R.B.: Probability and Measure Theory. Harcourt/Academic Press, Burlington (2000)

10. Athavale, A.: On the intertwining of joint isometries. J. Oper. Theory 23, 339-350 (1990)

11. Athavale, A.: On completely hyperexpansive operators. Proc. Am. Math. Soc. 124, 3745-3752 (1996)

12. Badea, C., Suciu, L.: The Cauchy dual and 2-isometric liftings of concave operators. J. Math. Anal. Appl. 472, 1458-1474 (2019)

13. Berg, C.: From discrete to absolutely continuous solutions of indeterminate moment problems. Arab. J. Math. Sci. 4, 1-18 (1998)

14. Berg, C., Christensen, J.P.R., Ressel, P.: Harmonic Analysis on Semigroups. Springer, Berlin (1984)

15. Birman, MSh, Solomjak, M.Z.: Spectral Theory of Selfadjoint Operators in Hilbert Space. D. Reidel Publishing Co., Dordrecht (1987)

16. Boas, R.P.: The Stieltjes moment problem for functions of bounded variation. Bull. Am. Math. Soc. 45, 399-404 (1939)

17. Chavan, S.: On operators Cauchy dual to 2-hyperexpansive operators. Proc. Edinb. Math. Soc. 50, 637-652 (2007)

18. Chō, M., Żelazko, W.: On geometric spectral radius of commuting $n$-tuples of operators. Hokkaido Math. J. 21, 251-258 (1992) 
19. Conway, J.B.: The Theory of Subnormal Operators. Mathematical Surveys Monographs, vol. 36. American Mathematical Society, Providence (1991)

20. Curto, R.E.: On the connectedness of invertible $n$-tuples. Indiana Univ. Math. J. 29, 393-406 (1980)

21. Curto, R.E.: Applications of several complex variables to multiparameter spectral theory. Surveys of Some Recent Results in Operator Theory, vol. II, pp. 25-90, Pitman Research Notes in Mathematics Series, 192. Longman Scientific \& Technical, Harlow (1988)

22. Curto, R.E., Lee, W.Y.: Towards a model theory for 2-hyponormal operators. Integr. Equ. Oper. Theory 44, 290-315 (2002)

23. Dickinson, D.R.: Operators; An Algebraic Synthesis. Macmillan, London (1967)

24. Douglas, R., Pearcy, C.: Hyperinvariant subspaces and transitive algebras. Mich. Math. J. 19, 1-12 (1972)

25. Duran, A.J.: The Stieltjes moments problem for rapidly decreasing functions. Proc. Am. Math. Soc. 107, 731-741 (1989)

26. Foguel, S.: A counterexample to a problem of Sz.-Nagy. Proc. Am. Math. Soc. 15, 788-790 (1964)

27. Furuta, T.: Invitation to Linear Operators. Taylor \& Francis, London (2001)

28. Gohberg, I., Goldberg, S., Kaashoek, M.A.: Classes of Linear Operators, Vol. I, Operator Theory: Advances and Applications, vol. 49. Birkhäuser Verlag, Basel (1990)

29. Gu, C.: On ( $m, p)$-expansive and $(m, p)$-contractive operators on Hilbert and Banach spaces. J. Math. Anal. Appl. 426, 893-916 (2015)

30. Halmos, P.R.: A Hilbert Space Problem Book. Springer, New York (1982)

31. Han, J.K., Lee, H.Y., Lee, W.Y.: Invertible completions of $2 \times 2$ upper triangular operator matrices. Proc. Am. Math. Soc. 128, 119-123 (2000)

32. Jabłoński, Z., Stochel, J.: Unbounded 2-hyperexpansive operators. Proc. Edinb. Math. Soc. 44, 613629 (2001)

33. Ji, K., Jiang, C., Keshari, D.K., Misra, G.: Rigidity of the flag structure for a class of Cowen-Douglas operators. J. Funct. Anal. 272, 2899-2932 (2017)

34. Jung, I.B., Ko, E., Pearcy, C.: Hyperinvariant subspaces for some $2 \times 2$ operator matrices. Kyungpook Math. J. 58, 489-494 (2018)

35. Jung, I.B., Ko, E., Pearcy, C.: Hyperinvariant subspaces for some $2 \times 2$ operator matrices, II. Kyungpook Math. J. 59, 225-231 (2019)

36. Kim, H.J.: Hyperinvariant subspaces for operators having a compact part. J. Math. Anal. Appl. 386, 110-114 (2012)

37. Lambert, A.: Subnormality and weighted shifts. J. Lond. Math. Soc. 14, 476-480 (1976)

38. Majdak, W., Mbekhta, M., Suciu, L.: Operators intertwining with isometries and Brownian parts of 2 -isometries. Linear Algebra Appl. 509, 168-190 (2016)

39. Müller, V.: Spectral Theory of Linear Operators and Spectral Systems in Banach Algebras. Operator Theory: Advances and Applications, vol. 139, 2nd edn. Birkhäuser, Basel (2007)

40. Pisier, G.: A polynomially bounded operator on Hilbert space which is not similar to a contraction. J. Am. Math. Soc. 10, 351-369 (1997)

41. Pólya, G.: Sur l'indétermination d'un problème voisin du problème des moments. C. R. Acad. Sci. Paris 207, 708-711 (1938)

42. Richter, S.: Invariant subspaces of the Dirichlet shift. J. Reine Angew. Math. 386, 205-220 (1988)

43. Rudin, W.: Real and Complex Analysis. McGraw-Hill, New York (1987)

44. Shimorin, S.: Wold-type decompositions and wandering subspaces for operators close to isometries. J. Reine Angew. Math. 531, 147-189 (2001)

45. Simon, B.: Operator Theory. A Comprehensive Course in Analysis, Part 4. American Mathematical Society, Providence (2015)

46. Stieltjes, T.: Recherches sur les fractions continues. Anns. Fac. Sci. Univ. Toulouse 8 (1894-1895), J1-J122; 9, A5-A47

47. Stochel, J.: Seminormality of operators from their tensor product. Proc. Am. Math. Soc. 124, 135-140 (1996)

48. Stochel, J., Stochel, J.B.: On the $\varkappa$ th root of a Stieltjes moment sequence. J. Math. Anal. Appl. 396, 786-800 (2012)

49. Sz.-Nagy, B., Foiaş, C.: Harmonic Analysis of Operators on Hilbert Space. Translated from the French and revised North-Holland Publishing Co., Amsterdam-London; American Elsevier Publishing Co., Inc., New York; Akadémiai Kiadó, Budapest (1970)

50. Taylor, J.L.: A joint spectrum for several commuting operators. J. Funct. Anal. 6, 172-191 (1970)

51. Taylor, J.L.: The analytic-functional calculus for several commuting operators. Acta Math. 125, 1-38 (1970) 
52. Vasilescu, F.-H.: Analytic Functional Calculus and Spectral Decompositions. Translated from the Romanian, Mathematics and its Applications (East European Series), 1., D. Reidel Publishing Co., Dordrecht; Editura Academiei Republicii Socialiste România, Bucharest (1982)

Publisher's Note Springer Nature remains neutral with regard to jurisdictional claims in published maps and institutional affiliations. 